\title{
EXPERIMENTAL STUDIES OF SLIGHTLY ENRICHED URANIUM, WATER MODERATED LATTICES \\ Part I. 0.600-in.-Diameter Rods
}

Herbert Kouts ANd Rudolph Sher

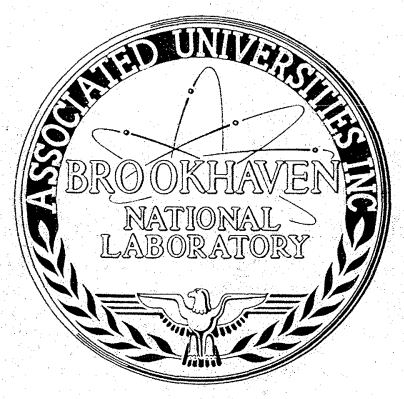

September 1957

BROOKHAVEN NATIONAL LABORATORY

Associated Universities, Inc. under contract with the United States Atomic Energy Commission 


\section{EXPERIMENTAL STUDIES OF SLIGHTLY ENRICHED URANIUM, WATER MODERATED LATTICES \\ Part I. 0.600-in.-Diameter Rods}

Herbert Kouts and Rudolph Sher

Work Done by
K.W. Downes
G.A. Price
A.P. Tierney
H. Kouts
R. SHER
V.J. WALSH

September 1957

BROOKHAVEN NATIONAL LABORATORY
Upton, N.Y. 


\section{E G A L N O T I C E}

This report was prepared as an account of Government sponsored work. Neither the United States, nor the Commission, nor any person acting on behalf of the Commission:

A. Makes any warranty or representation, express or implied, with respect to the accuracy, completeness, or usefulness of the information contained in this report, or that the use of any information, apparatus, method, or process disclosed in this report may not infringe privately owned rights; or

B. Assumes any liabilities with respect to the use of, or for damages resulting from the use of any information, apparatus, method, or process disclosed in this report.

As used in the above "person acting on behalf of the Commission" includes any employee or contractor of the Commission to the extent that such employee or contractor prepares, handles or distributes, or provides access to, any information pursuant to his employment or contract with the Commission.

\section{PRICE $\$ 1.25$}

Available from the Office of Technical Services,

Department of Commerce,

Washington 25, D.C. 


\section{Contents}

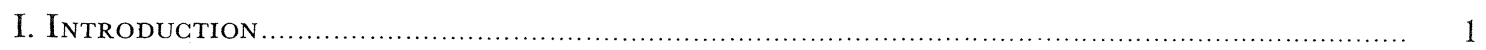

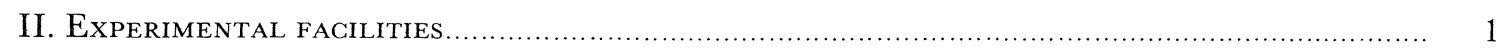

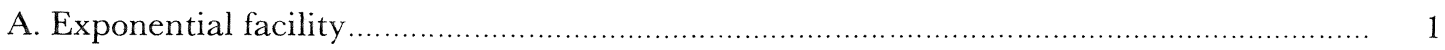

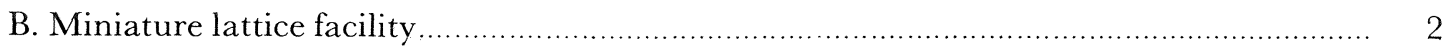

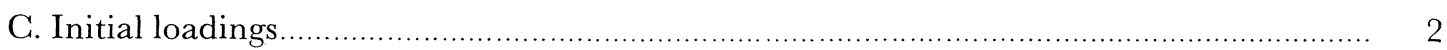

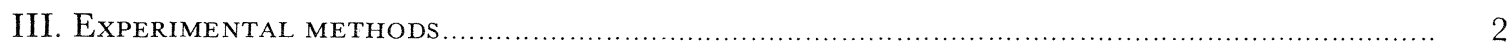

A. Axial flux traverses................................................................................................... 2

B. Radial flux traverses.................................................................................................. 4

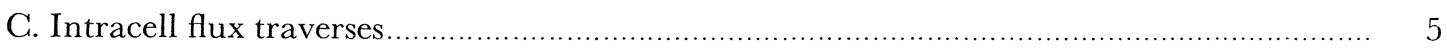

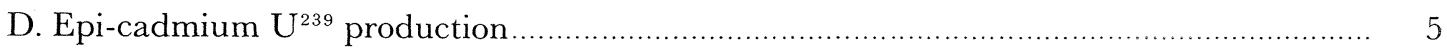

E. Gold cadmium ratio in fuel ............................................................................... 7

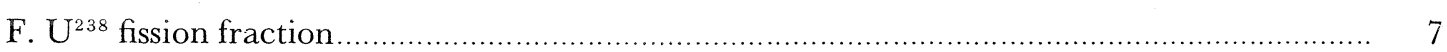

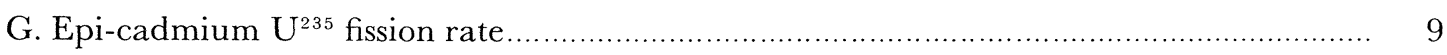

H. Boron-cadmium danger coefficients........................................................................... 9

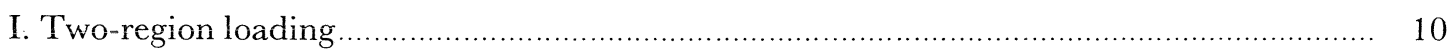

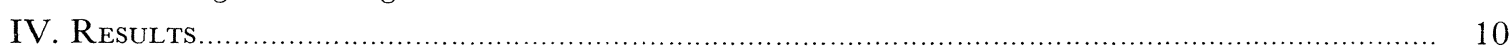

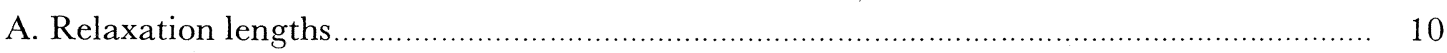

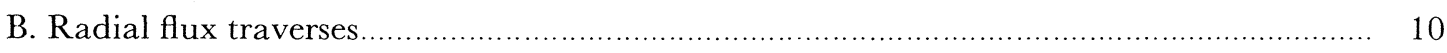

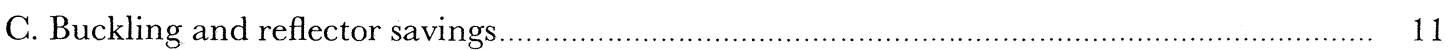

D. Intracell flux variation and thermal utilization............................................................... 12

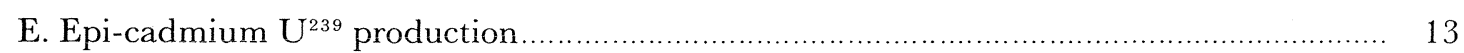

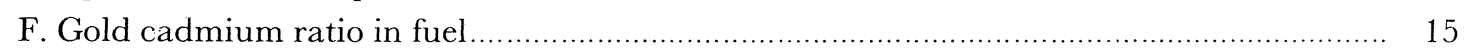

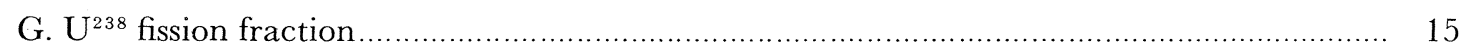

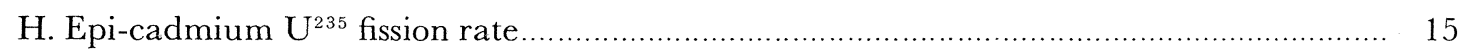

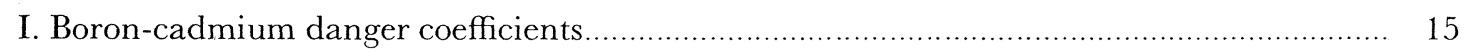

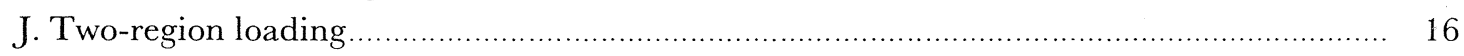

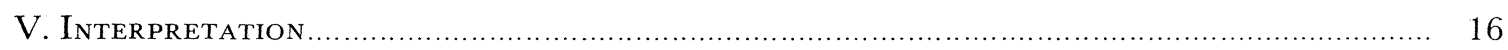

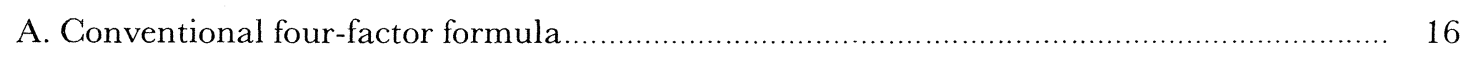

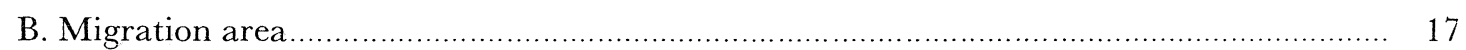

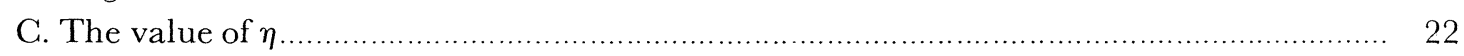

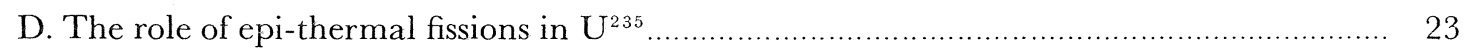

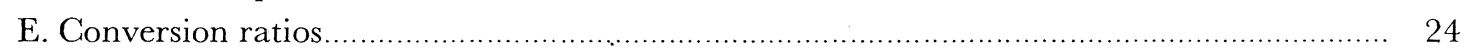

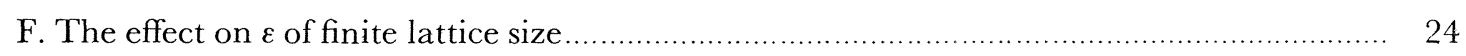

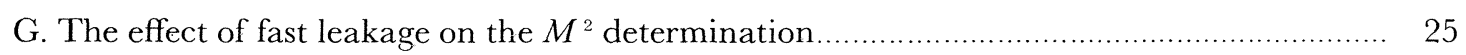

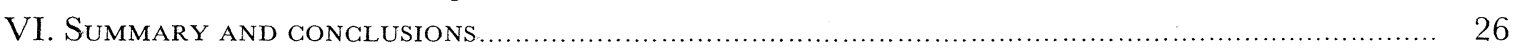

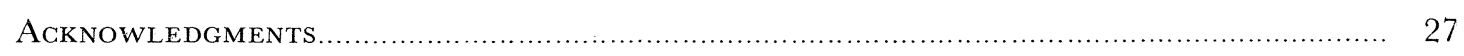

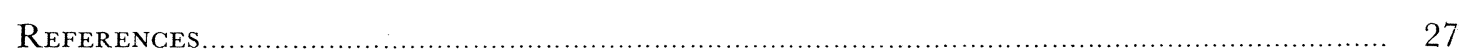

ApPENDIX I. Separation of uranium from its fission products by the sodium uranyl acetate method.. 28

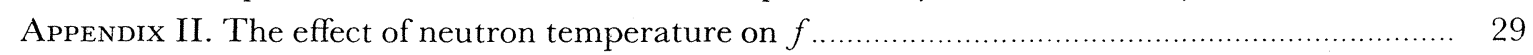

Appendix III. Subtraction of $1 / v$ contributions from epi-cadmium activations.......................... 32

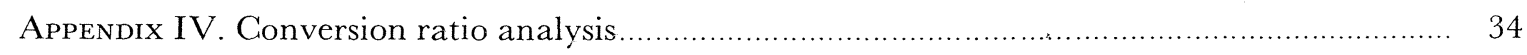

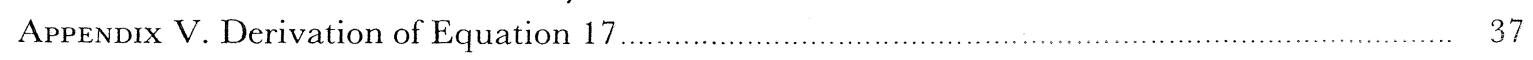

Appendix VI. Three-group analysis of fast leakage ............................................................. 38 


\section{EXPERIMENTAL STUDIES OF SLIGHTLY ENRICHED URANIUM, WATER MODERATED LATTICES \\ Part I. 0.600-in.-Diameter Rods}

\section{INTRODUCTION}

The experimental reactor physics program at Brookhaven has for the past few years been given mainly to a study of uranium-water lattices. The purpose of this undertaking has not been to provide direct design parameters for pressurized water reactor cores. Rather, it has been the intent to develop a body of experimental data which can be compared with the results of calculational schemes. Such work should help to improve the theoretical treatments to a point where design calculations of practical pressurized water reactor cores can be carried out with confidence.

The first phase of this programmatic study, devoted to the static properties of clean, cold, rodfueled assemblies, is nearly finished. This work has consisted of measurements of as many features of the neutron economy as possible, for a variety of fuel rod sizes and enrichments and lattice spacings.

The results of these experiments have been reported previously in internal memoranda and talks at meetings. Also, a fairly complete account of the measurements done with 0.600 -in. and 0.750 -in.-diameter rods was given at the Geneva conference in $1955 .^{1}$

In the course of collecting data for reports on other rod sizes, it has been decided, for several reasons, that these older results should be republished. Most of the quantities have been remeasured since 1955. The accuracy of some has thus been improved, and their values have changed slightly. Some systematic errors have been removed, notably from the $f$ measurement and from a few determinations of $B^{2}$. The resonance capture experiments, begun in 1954, had led to only tentative results by 1955 , and so were not reported in Geneva. These are now finished, and their implications must be included in any complete analysis of this work. Finally, a complete report on all BNL rod measurements, giving all final results in one place, ought to be useful. This is Part I of the final report.

Early reports gave results as interpreted through the four-factor formula. This practice is still followed here; but also the more basic data are presented in a form suitable for use with multigroup interpretations, because the design calculations for power reactors require a departure from fourfactor methods. Temperature coefficients, burnout, and stable fission product build-up all lead to neutron multiplication which varies from cell to cell. Furthermore, it has become standard practice to design nonuniformity into the reactor core at the beginning, for flux flattening, power flattening, or other engineering reasons (e.g., the PWR seed core). Therefore even heterogeneous cores are these days designed by multigroup methods.

On the other hand, there is a wide difference in the multigroup methods used at different laboratories. Therefore, although this report is directed toward use in connection with such theoretical methods, no attempt is made to carry out the connection here to any great extent.

The scope of Part I of this report is 15 lattices of 0.600 -in.-diameter uranium rods. Three fuel enrichments were used; these had nominal $\mathrm{U}^{235}$ contents of $1.0 \%, 1.15 \%$, and $1.3 \%$. Table 1 gives actual enrichments, atom densities, etc., for the fuel.

Five ratios of water-to-uranium volumes were studied with each fuel enrichment. Table 2 lists the pertinent geometrical data on these lattices.

\section{EXPERIMENTAL FACILITIES}

Three experimental facilities have been used in the course of these studies.

\section{A. Exponential Facility}

The first of these is an exponential experiment facility of reasonably standard design located on top of the Brookhaven graphite research reactor. 
The basic component is a water tank, $6 \mathrm{ft}$ in diameter and $6 \mathrm{ft}$ high, resting on a thermal column. At full pile power $(\approx 25 \mathrm{Mw})$, the entrant thermal neutron flux at the bottom of the tank is in the neighborhood of $10^{8} / \mathrm{cm}^{2}$-sec. The associated cadmium ratio as measured with 5-mil-thick indium is approximately $10^{5}$.

The lattices assembled in this tank consisted of cylindrical 4-ft-long rods canned in aluminum. The aluminum clad was 0.028 in. thick, and there was an air space of 0.005 in. between the uranium and the aluminum. Thus the aluminum tubing had an i.d. of $0.610 \mathrm{in}$. and an o.d. of $0.666 \mathrm{in}$. The rods were hung from the top, the weight being held by a support framework. Spacing to give the correct volume ratio was arranged by aluminum lattice plates at top and bottom, and by methyl methacrylate (Lucite) plates at 18-in. intervals between. The top of a typical lattice is shown in Figure 1.

Spacing tolerances were purposely set very low. Hole positions in the lattice plates did not deviate from design values by more than 0.002 in. hole-tohole, or by more than 0.020 in. between any pair of holes. Thus this fabrication error was insignificant.

Demineralized water was used at all times, except that occasionally the assemblies were poisoned with dissolved $\mathrm{H}_{3} \mathrm{BO}_{3}$.

Since the tank radius was $3 \mathrm{ft}$, whereas the radii of the uranium assemblies were in the neighborhood of $1 \mathrm{ft}$, all measurements were done with an effectively infinite water reflector on the sides.

\section{B. Miniature Lattice Facility}

Some small-sized assemblies (miniature lattices) were irradiated at high flux in a tunnel which runs under the Brookhaven reactor. The water tank in this case was $1 \mathrm{ft}$ in diameter and $2 \mathrm{ft}$ high. The fuel rods were $18 \mathrm{in}$. long, and were aluminum canned. A paraffin-filled box was used to reflect the assemblies in order to flatten the flux radially.

The neutron multiplication by a miniature lattice was of course very low. The incident leakage flux from the reactor was on the other hand quite high $\left(\sim 10^{9}\right)$, and the neutron levels within the loaded volumes were therefore large enough to allow measurements of fast fission fractions, resonance neutron absorption, detailed thermal neutron flux plots, and epi-cadmium fission fractions.
Table 1

Properties of the Uranium

\begin{tabular}{|c|c|c|c|c|}
\hline $\begin{array}{l}\text { Nominal } \\
\text { enrichment }\end{array}$ & $\begin{array}{c}\text { Actual } \\
\text { wt } \% U^{235}\end{array}$ & $\begin{array}{l}\text { Specific } \\
\text { gravity }\end{array}$ & $\begin{array}{c}n_{25} \\
\left(\text { atoms } / \mathrm{cm}^{3}\right)\end{array}$ & $\begin{array}{c}n_{28} \\
\left(\text { atoms } / \mathrm{cm}^{3}\right)\end{array}$ \\
\hline 1.3 & 1.299 & 18.898 & $6.288 \times 10^{20}$ & $4.719 \times 10^{22}$ \\
\hline 1.15 & 1.143 & 18.920 & $5.540 \times 10^{20}$ & $4.731 \times 10^{22}$ \\
\hline 1.0 & 1.027 & 18.898 & $4.971 \times 10^{20}$ & $4.732 \times 10^{22}$ \\
\hline \multicolumn{5}{|c|}{$\begin{array}{ll}\text { Diameter: } & 0.600 \pm 0.001 \text { in. } \\
\text { Length: } & 4 \mathrm{ft} \\
\text { Clad: } & 0.028 \text { in. } 2 \mathrm{~S} \text { aluminum } \\
\text { Air gap between fuel and clad: } 0.005 \text { in. nominal }\end{array}$} \\
\hline
\end{tabular}

Table 2

Geometrical Parameters of the Lattices

\begin{tabular}{|cccc}
\hline $\begin{array}{c}\text { Volume water } \\
\text { Volume uranium }\end{array}$ & $\begin{array}{c}\text { Distance between } \\
\text { rod centers (in.) }\end{array}$ & $\begin{array}{c}\text { Radius equivalent } \\
\text { cylindrical cell (in.) }\end{array}$ & $\begin{array}{c}\text { Volume \% } \\
\text { uranium }\end{array}$ \\
\hline 1. & 0.8537 & 0.4482 & 0.4480 \\
1.5 & 0.9444 & 0.4959 & 0.3660 \\
2 & 1.0273 & 0.5395 & 0.3092 \\
3 & 1.1754 & 0.6171 & 0.2363 \\
4 & 1.3070 & 0.6862 & 0.1911 \\
\hline
\end{tabular}

Figure 2 shows a miniature lattice in position in the tunnel, ready to be covered and moved under the reactor.

\section{Initial Loadings}

Considerations of safety made it imperative that the initial loading of each lattice be treated as a critical assembly. These loadings were performed in a tank at the critical assembly facility, and each was accompanied by the standard safety practices established for critical approaches. Although no lattice was actually taken to a critical size, the associated instrumentation and safety systems were sufficient for critical assembly operations.

This facility was also used in connection with measurements of the moderator neutron temperature described in section III-H.

\section{EXPERIMENTAL METHODS}

\section{A. Axial Flux Traverses}

These measurements were carried out in the exponential experiment facility mentioned in section II-A. Typically, a lattice was loaded to at 


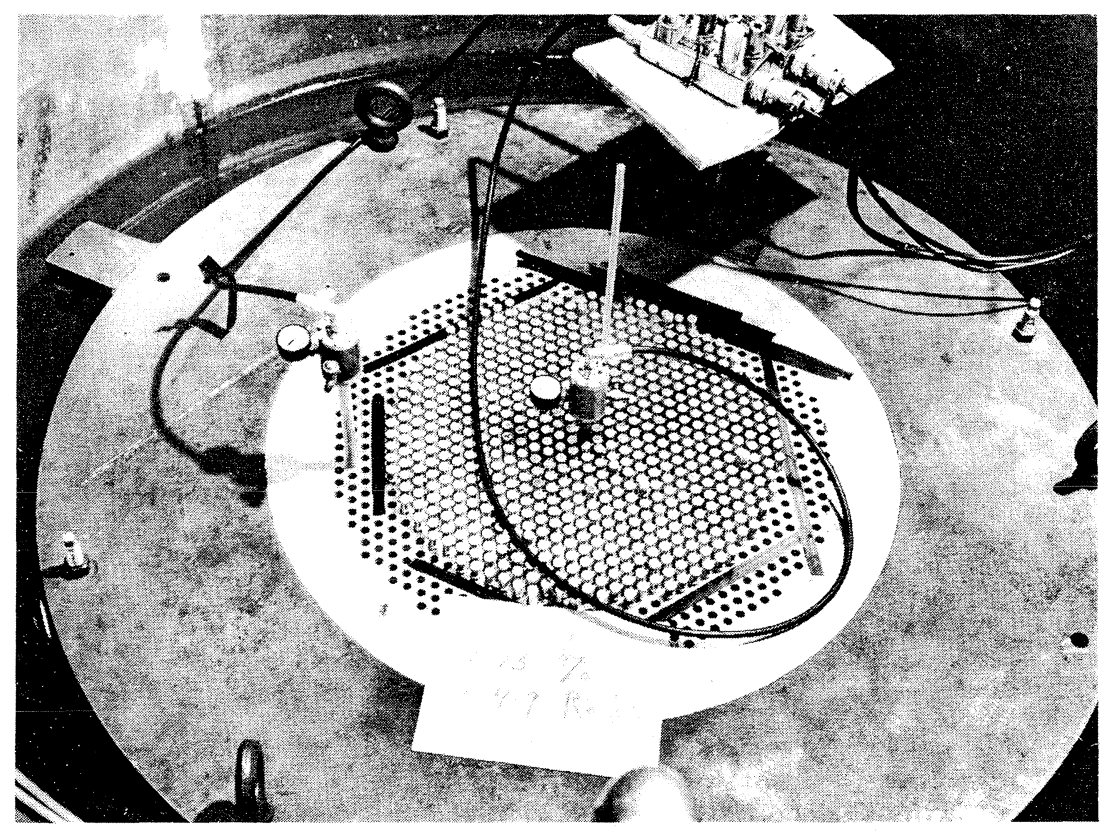

Figure 1. Exponential assembly.

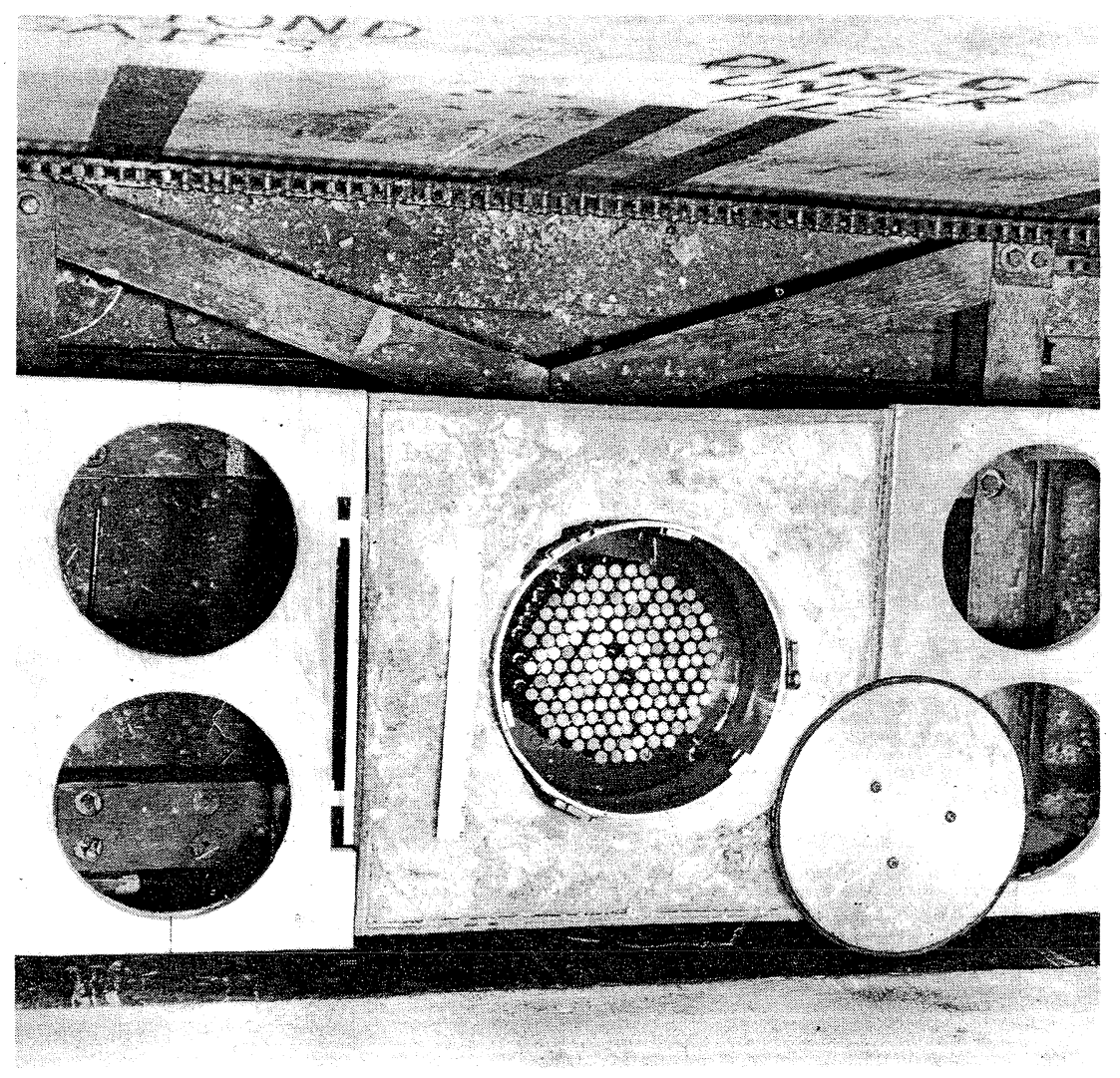

Figure 2. Miniature lattice. 
most about $80 \%$ of critical mass $\left(k_{\text {ef }} \approx 0.97\right)$, and was subjected in the water tank to the thermal neutron source from below.

The axial flux variation was found by indium foil activation. Identical foils were set at $10-\mathrm{cm}$ intervals in a Lucite rod, which was then inserted through the top lattice plate. During activation, then, these foils lay in a vertical line which passed through the center of a triangular lattice cell whose corners were three neighboring fuel elements. The spacing of fuel in the $1: 1$ volume ratio lattices was, however, too close to allow insertion of a Lucite foil rod. In these cases, the relaxation lengths were found from the activation of indium foils placed between sections of a cut-up fuel element.

The indium foils in the Lucite rods were fixed with their surfaces parallel to the axial flux gradient. This geometry served to minimize the difference in activity of the two faces.

All foils were $\beta$-counted with end-window Geiger counters. Each activity was measured in six separate counters, each side of the foil being observed a total of three times. The total count per foil was at least 10,000, so that the statistical accuracy per point was $1 \%$. The indium thickness was 0.010 in.; it had previously been established that the curve of foil sensitivity vs thickness had a broad maximum here, and therefore no corrections had to be made for foil weight. In spite of this, foil weights were matched in individual runs.

Since all foils used in a given traverse had identical exposure periods, it was only necessary to reduce the activities to those at the time counting actually was begun.

\section{B. Radial Flux Traverses}

Traverses were made to find the variation of neutron flux with distance from the central axis of exponential assemblies (the height being fixed). The spacial distribution sought in these instances was the cell-to-cell variation, with the intracell variation having been factored out. Values on this macroscopic curve could be found by sampling the neutron flux at those points along a radius which are equivalent from the standpoint of local flux depression by fuel. For a number of reasons it was decided best to choose these equivalent points in the fuel rods rather than in the water.

Two techniques were used, both involving the use of a special fuel rod which replaced in turn the individual rods along a chosen radius. In one case, the special rod was simply a fuel element sectioned to contain an indium foil. In the other case a fuel element was used containing a fission chamber in a centrally drilled cavity. It was necessary in both cases to use a monitor to account for pile power variations, because the individual points on a given radial flux traverse were not measured at the same time. When the foil technique was used to find the flux plot, the monitor was a second sectioned fuel element containing an indium foil, exposed simultaneously with the first but at a different and fixed lattice position. The monitor for the fission counter measurements was a second fission counter clamped in place outside the lattice. The lattice shown in Figure 1 contains these two fission chambers.

Because of the basic hexagonal symmetry of the lattices, the choice of radii for radial flux measurements required a certain amount of care. An effort was made to achieve as nearly a cylindrical loading as possible (see Figure 1), but even in the best of circumstances the radial distribution would not be the same in different directions. Therefore radial distributions were measured in each case along six lines, in a symmetrical manner. Three of these lines were along the diagonals of the hexagonal pattern; these are called "straight" radials because they contained a straight line of fuel rods. The other three were lines normal to the faces of the hexagon, and since only a broken

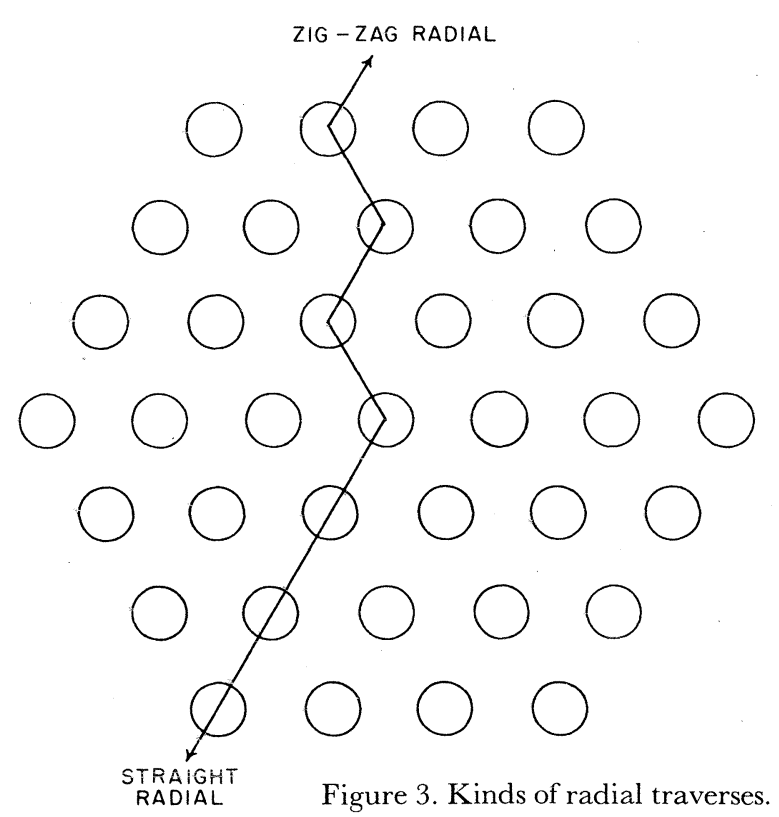


line of fuel elements lay in these directions, the radials are called "zig-zag." The geometry of both radial lines is shown in Figure 3.

\section{Intracell Flux Traverses}

The thermal utilization depends among other things on the way the thermal neutron flux varies with position in an individual lattice cell. This variation was therefore measured.

The flux detectors used were small foils made of a mixture of dysprosium oxide and polyethylene. Dy ${ }^{164}$ has a thermal neutron cross section of approximately 7000 barns, and is $28 \%$ abundant in normal dysprosium. $D y^{165}$ is a $\beta$-emitter, with a half-life of $2.32 \mathrm{hr}$. The near-thermal resonances of dysprosium are all associated with other isotopes, ${ }^{2}$ and activation experiments within the lattice assemblies revealed a cadmium ratio of approximately 100 for the 2.32 -hr activity. These features - high cross section, moderate half-life, and low epi-cadmium activation - made dysprosium uniquely suited for use in intracell flux measurements.

The foils used were $1 / 16$ in. in diameter and 0.010 in. thick, and contained $50 \% \mathrm{Dy}_{2} \mathrm{O}_{3}$ by weight. Decay curves showed no sensible impurity content giving rise to other $\beta$-activities than the one of interest. Since the sensitivity per foil varied, it was necessary to perform intercalibrations, and these were accomplished by exposing foils at equal radii on a revolving wheel in a thermal neutron flux. The reciprocals of saturated activities from such uniform exposure histories are (proportional to) the necessary intercalibration factors.

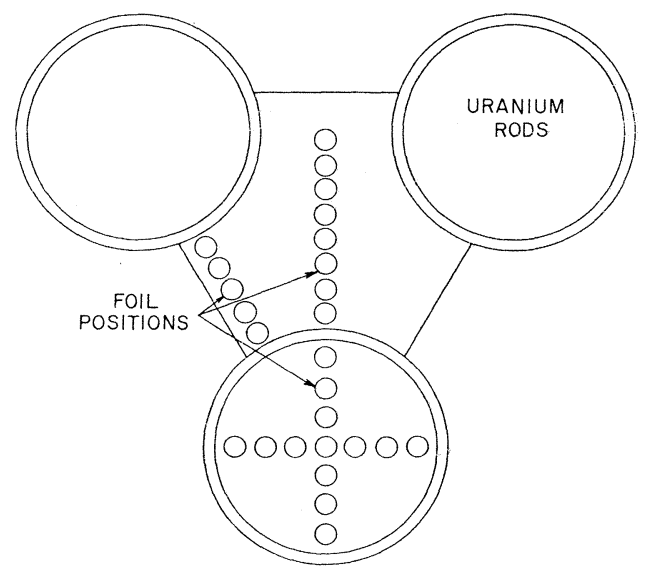

Figure 4. Foil positions for $2: 1$ lattice.
An intracell flux traverse consisted of the simultaneous exposure of sets of foils in a uranium rod and in the neighboring water moderator. Those foils exposed in the fuel were placed in small holes milled in a cross section cut of a rod. Those in the water were held in place in a Lucite sheet $1 / 32$ in. thick, shaped to fit snugly against three neighboring fuel rods. These features are apparent in Figure 4, which also shows the lines along which the variation of flux was measured. Two crossed diameters in the fuel were used, and also the two principal directions in the moderator.

The counting techniques used with dysprosium were the same as those used with indium.

Intracell flux traverse measurements were performed with miniature lattices of uranium rods and water (see section II-B). A series of comparison measurements in these and in full-sized exponential assemblies had earlier established that miniature lattice methods led to correct flux plots.

Because of the small size of miniature lattices, it was necessary to correct all foil activities by a factor $J_{0}^{-1}(a r / R)$ where $R$ is the effective lattice radius and $r$ the distance of the foil from the center. This factor however was never smaller than 0.99 , and so any error introduced by the uncertain knowledge of $R$ was negligible.

\section{Epi-Cadmium Uranium-239 Production}

These measurements were designed to yield the ratio of the epi-Cd capture rate in $\mathrm{U}^{238}$ in a fuel rod to the thermal (sub-Cd) capture rate. Natural $\mathrm{U}$ foils, approximately $0.005 \mathrm{in}$. thick, and of the diameter of the fuel rod, were irradiated in the fuel. One foil was "bare"; the other, together with a small amount of fuel material (buttons) on either

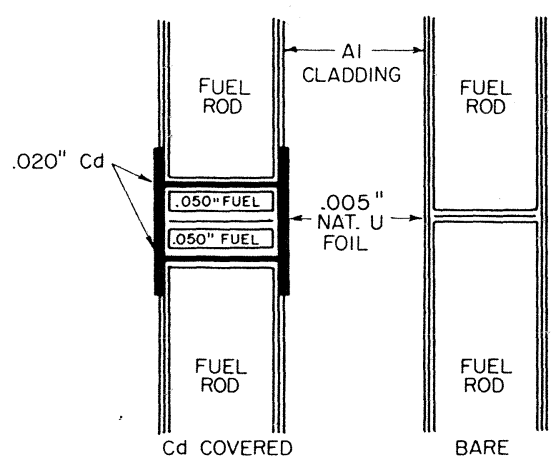

Figure 5. Fuel rod assemblies of bare and Cd-covered foils. 


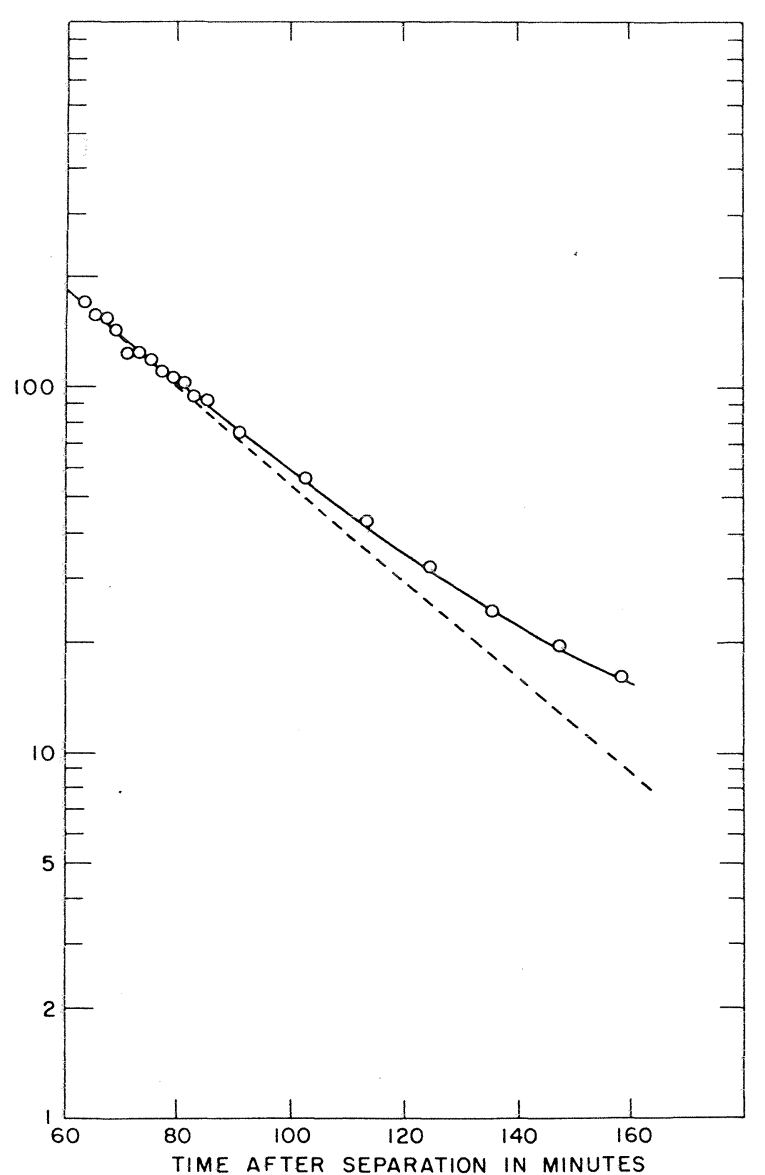

Figure 6. Decay of $\mathrm{U}^{239}-\mathrm{Np}^{239}$ sample.

side of it, was surrounded by a sleeve and end pieces of approximately 0.020 -in. Cd (Figure 5). The actual thickness of the sleeve was about 0.015 in.; of one end piece, $0.017 \mathrm{in}$.; and of the other end piece, $0.022 \mathrm{in}$. The fuel buttons were 0.050 in. thick, and, by minimizing the effects of streaming through the Cd end pieces, ensured that the resonance neutron spectrum entering the Cdshielded foil was typical of that entering any section of fuel.

The foils were placed at symmetric positions in miniature lattices and irradiated for $40 \mathrm{~min}$. After irradiation the fuel rods containing the foils were dismantled and the foils recovered. The uranium was chemically separated (as sodium uranyl acetate) from fission products and its daughter activities. The chemical procedure (given in Appendix I) takes about $1 \mathrm{hr}$. Three aluminum planchets containing from 1 to $15 \mathrm{mg}$ each of sodium uranyl acetate were prepared from

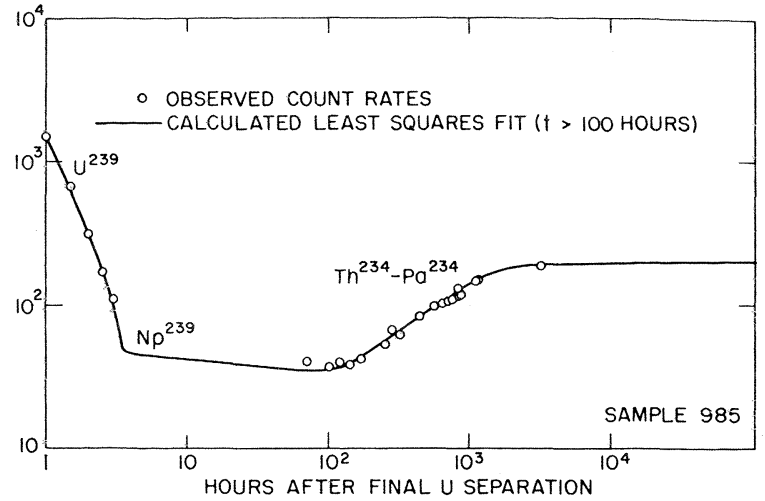

Figure 7. Growth of $\mathrm{Th}^{234}$ and $\mathrm{Pa}^{234}$ activity.

each foil and when dry were counted in end-window Geiger counters. The $\beta$-decay curve of the resulting activity consists of 1 ) the 23.5 -min activity of $\left.\mathrm{U}^{239}, 2\right)$ the 2.3 -day activity of $\mathrm{Np}^{239}$ which grows in as the $\mathrm{U}^{239}$ decays, 3 ) the $\mathrm{Pu}^{239}$ activity, which is completely negligible because of its long half-life, and 4) the $\mathrm{Th}^{234}$ and $\mathrm{Pa}^{234}$ daughter activity of $\mathrm{U}^{238}$, which grows at a rate nearly corresponding to the 24.1-day half-life of $\mathrm{Th}^{234}$. Of these components, the $\mathrm{U}^{239}$ and $\mathrm{Np}^{239}$ activities depend only on the a mount of $U^{239}$ present and are the only activities observed in the first few hours after the irradiation and separation. The $\mathrm{Th}^{234}$ and $\mathrm{Pa}^{234}$ activities depend only on the a mount of $\mathrm{U}^{238}$ in the sample, and are the only activities observed long after the separation, after the 2.3-day $\mathrm{Np}^{239}$ has decayed out. Thus the ratio of $\mathrm{U}^{239}$ (or $\mathrm{Np}^{239}$ ) activity to $\mathrm{Th}^{234}$ activity is a measure of the specific $\mathrm{U}^{239}$ production. Since the bare and Cd-covered foil samples were irradiated, separated, and counted together, the $\mathrm{U}^{239}$ / $\mathrm{Th}^{234}$ activity ratio for the bare foil was proportional to the sub-Cd plus epi-Cd capture rate in $\mathrm{U}^{239}$, while the corresponding ratio for the Cdcovered sample was proportional to only the epiCd capture rate. From these, the ratio of epi-Cd to sub-Cd capture could easily be obtained.

In practice the $\mathrm{U}^{239}$ decay was followed long enough (approximately $3 \mathrm{hr}$ ) for the $\mathrm{Np}^{239}$ activity to grow in appreciably. Figure 6 shows the composite decay, together with a calculated $U^{239}$ $\mathrm{Np}^{239}$ decay curve. Figure 7 shows the subsequent growth of the daughter activities of $\mathrm{U}^{238}$. From these curves it is incidentally evident that the separation adequately removes fission product activity. 
In addition to assaying the amount of $U^{238}$ in each sample by counting its daughter activity some 3 weeks after irradiation, two other methods were used: first, the sodium uranyl acetate was weighed in the planchets, and second, the samples were quantitatively analyzed for uranium by R.W. Stoenner of the BNL Chemistry Department. All three methods gave results which generally agreed to within $5 \%$.

The ratio of specific $\mathrm{U}^{239}$ activities in the bare and shielded foils is designated as $R_{u}$ and the ratio of epi-Cd to sub-Cd capture as $S$, thus $S=\left(R_{u}-1\right)^{-1}$.

It should be noted that these measurements were done at BNL only on the $1.0 \% \mathrm{U}^{235}$ enrichment.

\section{E. Gold Cadmium Ratio in Fuel}

One quantity of interest in certain interpretations of measurements relating to the resonance escape probability is the Cd ratio of thin gold foils in the fuel. Measurement of this consisted simply of irradiating 0.0005-in.-thick Au foils in place of the uranium foils described in the preceding section (with one difference: in some of the runs the 0.050 -in. fuel buttons within the Cd pill-box were omitted). The $\mathrm{Au}^{198} \beta$-activities were counted in end-window Geiger counters, and the Cd ratios so obtained were corrected to zero thickness by means of a factor determined in a set of similar measurements in the BNL reactor on a series of $\mathrm{Au}$ foils of different thicknesses and $\mathrm{Pb}-\mathrm{Au}$ alloy foils (to simulate extremely thin foils).

In the BNL reactor work, it had been determined that the zero-thickness ratio of epi-Cd activation in Au to sub-Cd activation was 2.32 times the ratio measured in 0.0005 -in.-thick foils. Stated another way, the zero-thickness Cd ratio, $R_{0}$, is related to the 0.0005 -in.-Cd ratio, $R$, by

$$
R_{0}=1+\frac{R-1}{2.32} \text {. }
$$

\section{F. Uranium-238 Fission Fraction}

These measurements are based upon fission catcher techniques. Since the time of the Geneva report, the measurements have been repeated in miniature lattices and extended to all the 0.600-in. lattices. It was found that lack of reproducibility in some of the measurements could be eliminated if the $99.0 \%$ pure commercial $\mathrm{Al}$ foil used for the catchers were replaced by high purity (99.8\%) Al.

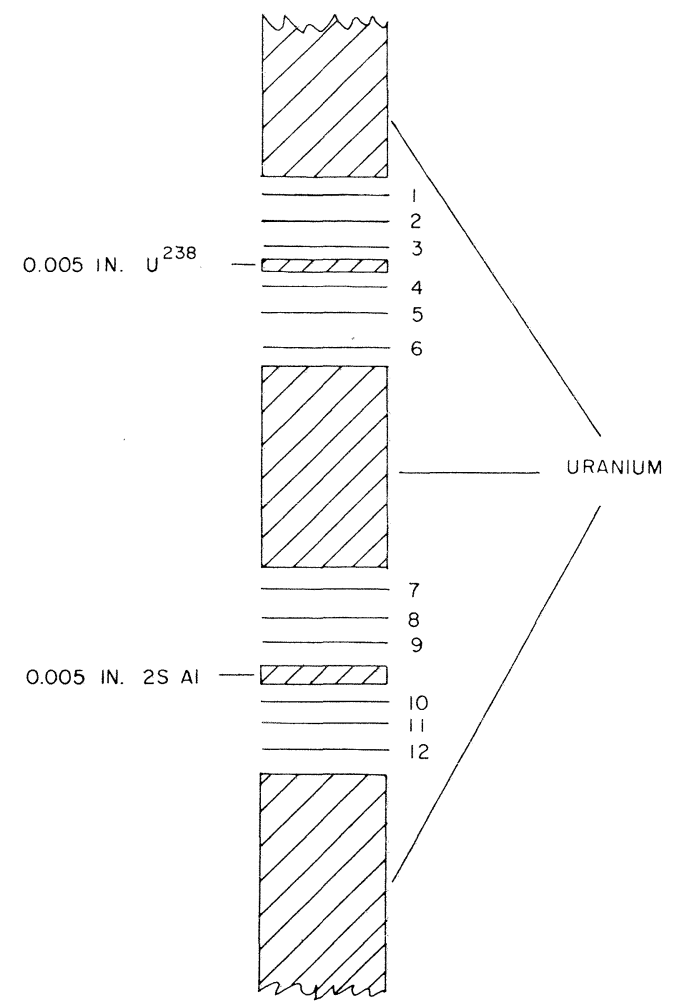

Figure 8. Catcher foil arrangement in measuring $F$.

All the miniature lattice measurements therefore were done with the new foils.

The measurements yield the ratio of the fission rate in $U^{238}$ in the fuel to the fission rate in $U^{235}$. This ratio, $F$, was determined in the following way.

A fuel rod was cut crosswise into sections, and between these were placed sandwiches of aluminum and uranium foils. The aluminum foils served to capture fission products from the uranium, and the activities of these fission products led to a measure of the fission density in the uranium.

Two such sandwiches were used per measurement of $F$. One consisted of three layers of 0.001in.-thick aluminum on each side of a depleted uranium foil, this entire sandwich being compressed between two sections of the fuel rod. The depleted uranium foil contained only $3 \times 10^{-6}$ parts of $U^{235}$ per part of $U^{238}$. In the neutron flux of the exponential experiments, the $U^{235}$ fission rate in the foil was less than $1 \%$ of the $U^{238}$ fission rate; accordingly any fission occurring in it could be attributed to $: \mathrm{U}^{238}$ alone. The second foil sandwich was precisely the same as the first, except that the depleted uranium was replaced by an 
aluminum foil, 0.005 in. thick. The two sandwiches were separated by a section of uranium rod 2 in. long. The experimental arrangement of fuel rod and aluminum and uranium foils is shown in Figure 8.

It was assumed that the fission densities in the section of fuel rod used were the same as if the uranium had not been cut and the foil sandwiches inserted. Actually, the foil sandwiches constituted a low absorption region through which thermal neutrons could stream; this effect increased the thermal fission rates locally. A simple calculation showed that this streaming could have altered the measured values of $F$ by at most about $0.1 \%$.

The fuel rod used was inserted in the center of a miniature lattice, replacing a standard uranium rod. The entire lattice was then irradiated for about $10 \mathrm{~min}$. At the end of this time the rod was removed, and the activities of the foils designated in Figure 8 as 1, 3, 4, 6, 7, 9, 10, and 12 were determined with end-window $\beta$-ray counters.

In a measurement of this sort, the fission product activities of foils 1, 6, 7, and 12 are proportional to the local fission density in the fuel rod. The fission product activities of foils 3 and 4 are proportional to the fission density in the depleted uranium foil. Since the range of fission products in aluminum is approximately 0.0004 in., one catcher is sufficient to stop the fission products emitted by the uranium with which it is in contact.

The activities of foils 9 and 10 give the background which must be subtracted from all other foil count rates. These decay rates are caused by the thermal activation of impurities, and by $(n, p)$, $(n, 2 n)$ and $(n, \alpha)$ reactions in the aluminum. Actually, the two foil sand wiches were not located at the same height in the lattice, and so were not ex-

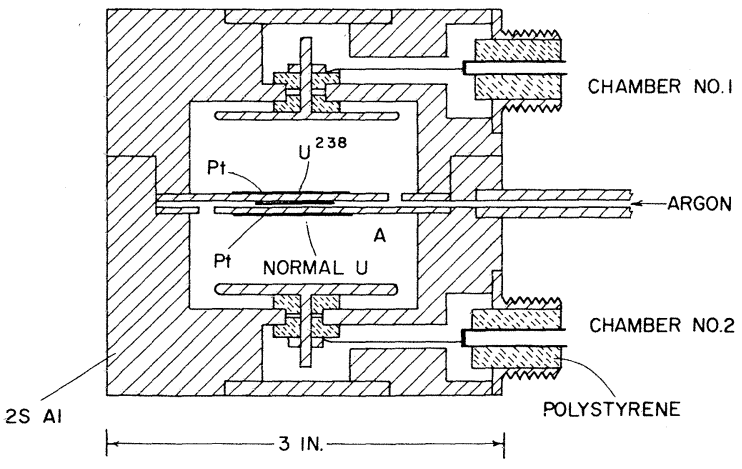

Figure 9. Double fission chamber. posed to the same neutron flux levels. Therefore, before the background correction given by foils 9 and 10 could be applied, it had to be multiplied by the ratio of flux densities at the heights of the two sandwiches.

The determination of $F$ from the foil-counting was carried out as follows. Catcher foils 1, 3, 4, 6, 9 , and 10 were counted simultaneously, being rotated about among the counters so that each foil was counted the same number of times in each counter. At the same time, foils 7 and 12 were counted in a separate pair of counters to give a decay curve for the fission products. This curve was used to correct the activities of the catcher foils back to the beginning of the foil-counting, called time $t=0$. (Normally foil-counting began $30 \mathrm{~min}$ after the end of the exposure; this permitted the short-lived activity from radiative capture in the aluminum to die out.)

The backgrounds as determined from foils 9 and 10 were subtracted from the catcher foil activities, and the activities of foils 1, 3, 4, and 6 were corrected to $t=0$ by means of the decay curve measured with foils 7 and 12. The corrected activities for each foil were then summed for all counters. Finally, these sums were added for foils 1 and 6 to obtain a quantity proportional to the fission density in the fuel rod, and were summed for foils 3 and 4 to get a number proportional to the fission density in the depleted uranium. This latter number when corrected for the slightly different atomic densities in the depleted uranium and the fuel rod was proportional to the fission density in $\mathrm{U}^{238}$ in the rod. Thus

$$
\begin{aligned}
& f_{25}=C_{1}\left(R_{1}-R_{2}\right), \\
& f_{28}=C_{2} R_{2}
\end{aligned}
$$

where $R_{1}$ and $R_{2}$ are respectively the final corrected decay rates for foils 1,6 , and for foils 3,4 , the latter corrected for $\mathrm{U}^{238}$ density; $f_{25}$ and $f_{28}$ are respectively the fission densities locally in the $\mathrm{U}^{235}$ and the $\mathrm{U}^{238}$ in the fuel rod; and $C_{1}$ and $C_{2}$ are the constants of proportionality, which would be equal except that the decay curves of fission products from $\mathrm{U}^{238}$ and $\mathrm{U}^{235}$ are not precisely the same. Then from Equation (3),

with

$$
f_{28} / f_{25}=F=K R_{2} /\left(R_{1}-R_{2}\right)
$$

$$
K=C_{2} / C_{1} .
$$




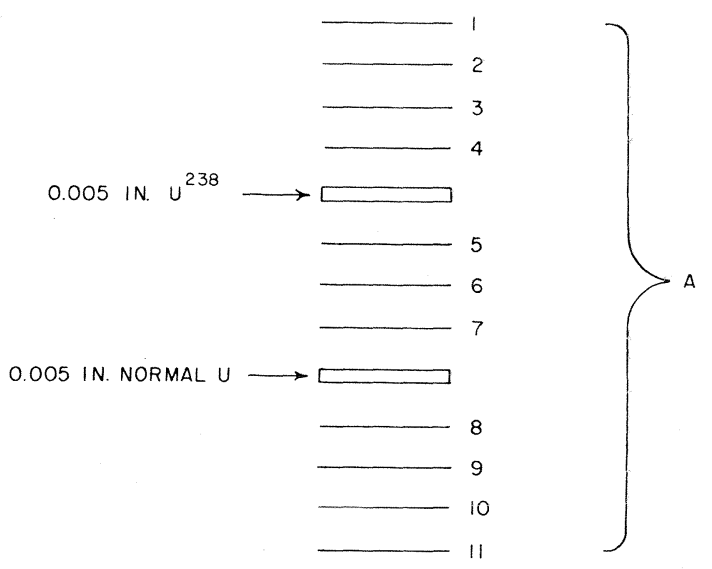

Figure 10. Foil sandwiches for $F$ calibration.

The quantity $K$ depends upon the exposure time and the period of time during which counting took place. A separate calibration experiment was necessary to determine it.

A "double" fission chamber was constructed, having the design shown in Figure 9. The cathode of each half of the chamber had on it a carefully weighed amount of uranyl nitrate, deposited in solution and then dried. One cathode was sensitized with a sample of the depleted uranium used in fast effect measurements; the other had natural uranium. In each case the layer of uranyl nitrate was thin compared with the path length of fission products, so that each fission on a cathode led to a count in its half of the chamber.

A sandwich of aluminum catcher foils with depleted and normal uranium foils was placed in the chamber; the arrangement of this sandwich is shown in Figure 10.

The chamber was then exposed to a mixture of fast and thermal neutrons of approximately the same character as that observed in one of the assemblies. The fission rates in the two halves of the fission chamber were recorded, and the catcher foils were afterwards removed and counted in the same way as for a fast effect measurement. The ratio of fission chamber count rates led to the ratio of $\mathrm{U}^{235}$ fission rates to $\mathrm{U}^{238}$ fission rates in the flux used, and the catcher foil activities led to the ratio of associated decay rates.

The equivalence of the two halves of the fission chamber was tested by interchanging the cathodes. The subsequent change in ratio of count rates was $0.3 \%$, which was below the probable error from the counting statistics.
As a result of the calibration experiment, the value of $K$ for the exposure and counting schedules used could be determined. In general $K$ was within a few percent of unity.

\section{G. Epi-Cadmium Uranium-235 Fission Rate}

In these measurements the Cd ratio of $\mathrm{U}^{235}$ fissions in the fuel was determined. High purity Al catcher foils were used. The "bare" foils were in contact with the ends of split sections of fuel rod. The Cd-shielded catchers were in contact with the fuel buttons in the Cd pill-box described in section III-D. Since the Al background would be expected to be different within the pill-box and outside it, separate sets of background foils were included in the foil assemblies. The catcher activities from the shielded foil and the bare foil were then corrected for $\mathrm{Al}$ background and fast fissions in $\mathrm{U}^{238}$ (as determined from the results of the fast fission measurements) to get the Cd ratio of $U^{235}$ fissions.

\section{H. Boron-Cadmium Danger Coefficients ${ }^{3}$}

The relative danger coefficients of boron and cadmium in solution in the moderator water were found for one lattice of $1.15 \%$ rods at a volume ratio of $3: 1$. This assembly was taken nearly to critical ( $k_{\text {ef }} \approx 0.998$ ), and the neutron level from multiplication of spontaneous fission neutrons was observed. A small amount of boric acid solution was added to the moderator, and the new neutron level was measured. A small amount of dissolved cadmium sulfate was then added, and the measurement repeated.

Three counters were used to measure the flux level at each stage. One was a small $\mathrm{BF}_{3}$ counter at the center of a triangular lattice cell, the second was a fission counter similarly placed, and the third was a fission counter in a fuel rod (one which had been made for radial flux traverses). Since the assembly was so near critical, the harmonic content of the flux inside the loaded volume was very small, and therefore the observed count rates from the three detectors were reliable measures of neutron multiplication.

The boron and cadmium contents of the moderator were later determined by chemical analysis.

While the measurements of flux level were in progress, the water temperature was monitored by means of a chromel-alumel thermocouple. 


\section{Two-Region Loading ${ }^{4}$}

One measurement was made of the amount by which the critical size changed when the central region of a lattice of $1.15 \%$ enriched rods was replaced by $1.0 \%$ fuel. This experiment was performed at a volume ratio of $3: 1$.

The critical size was determined from extrapolation of the critical approach curve to infinite flux level, with the neutron source being simply that arising from the spontaneous fissions. Two critical approaches were made, one with a uniform lattice of $1.15 \%$ enriched elements, and one with the inner 19 rods replaced. Thus two values of critical size were obtained.

One neutron detector was used, a small $\mathrm{BF}_{3}$ counter near the lattice center. Calculations of harmonic content of the flux at the high neutron multiplications involved implied that, although the amplitudes of individual harmonics were as much as $1 \%$ of the fundamental amplitude, the sum of harmonics did not exceed $0.01 \%$ of the fundamental. Thus the critical approach curve was an accurate measure of the behavior of neutron multiplication.

\section{RESULTS}

\section{A. Relaxation Lengths}

Measurements were made at all five volume ratios of moderator to fuel, and for all three enrichments. A large number of the assemblies made up of $1.3 \%$ and $1.15 \%$ enriched uranium were poisoned with $\mathrm{H}_{3} \mathrm{BO}_{3}$ in solution in the moderator water. Relaxation lengths were measured in these instances also.

The data reported break up naturally into two types: relaxation lengths obtained at each of a number of fuel rod loadings, and several relaxation lengths found only at the maximum fuel loading. This division is a consequence of the two ways used to infer the buckling.

The quantities measured in a determination of the relaxation length $L$ are relative neutron fluxes $A_{i}$ at several distances $Z_{i}$ from the bottom of the uranium. These must be fitted to the function

$$
A=C \sinh \left[m\left(Z_{0}-Z\right)\right]
$$

in a manner such that $m=L^{-1}$ can be found. The general problem then is one of performing a fit of the measured pairs $A_{i}, Z_{i}$ to the function in Equa- tion (6), the criterion of least squares establishing values of $C, m$, and $Z_{0}$.

In these experiments the solution was carried out in several stages. $Z_{0}$ (the extrapolated end point above the lattice) was held as constant as possible over the course of the experiments by the maintenance of a fixed water level. At time intervals dictated by needs, special eleven-point axial flux traverses were made and subjected to threeconstant least-squares fits; the value of $Z_{0}$ so found was then used for other relaxation length measurements done in between times.

The ordinary axial flux traverses were based on activation of six foils at $10-\mathrm{cm}$ intervals. The activities were plotted on semilog graph paper, and a first guess, $m_{1}$, at the value of $m$ was made. Each foil activity was multiplied by the function

$$
\frac{\exp \left[m_{1}\left(Z_{0}-Z_{i}\right)\right]}{\sinh \left[m_{1}\left(Z_{0}-Z_{i}\right)\right]}
$$

which was always very near unity and never exceeded 1.08, and the converted values $A_{i}{ }^{\prime}$ of foil activity were then least-squares fitted to an exponential function. The relaxation constant $m_{2}$ found in this fashion was used to recalculate the factor in Equation (7), and the process was iterated until the value of $m$ had converged. Such a procedure was considerably more amenable to desk calculation than fitting to a sinh function would have been.

\section{B. Radial Flux Traverses}

The representative form of a radial flux traverse in these assemblies can be observed from Figure 11. There was evident flux peaking in the neighborhood of the core-reflector interface, and so the full curve inside the core would have had to conform to a function such as

$$
C_{1} J_{0}\left(B_{r} r\right)+C_{2} I_{0}(\mu r)
$$

where $C_{1}, C_{2}, B_{r}$, and $\mu$ are constants, and $\mathrm{B}_{r}{ }^{2}$ is the radial buckling. The quantity of interest to be deduced from a radial flux traverse is $B_{r}$; because of the reflector effect represented by the second term of Equation (8), care had to be exercised in performing the analysis.

The precaution finally decided upon was simply the rejection from the analysis for $B_{r}$ of all measurements taken within three lattice units of the interface. It was supposed that inside this limit 


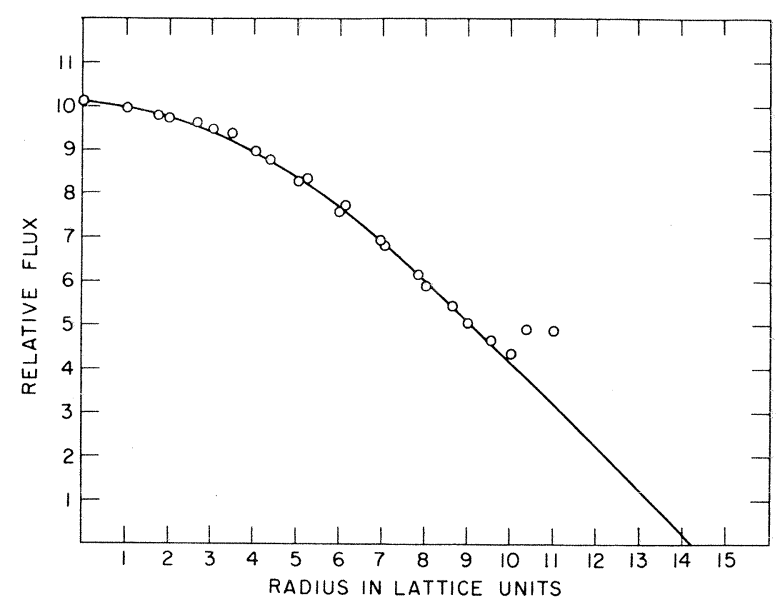

Figure 11. Composite radial traverse.

the flux contained only the core component $J_{0}$, and that a least-squares fit of the data to

$$
C_{1} J_{0}\left(B_{r} r\right)
$$

would yield correct values of $C_{1}$ and $B_{r}$.

Two steps were taken to corroborate the above assumption. The first consisted of recalculating some least-squares fits when also the fourth lattice point from the boundary was omitted, and comparing results. The second test involved inferring $C_{2}$ and $\mu$ from the shape of the curve near the interface and comparing $\mu$ with the value it should have according to two-group theory. Each test led to consistency, which confirmed the basis of the analytical methods.

The quantity $B_{r}$ has the form

$$
B_{r}=a_{1} /(R+\lambda), \quad a_{1}=2.4048 \ldots
$$

with $R$ being the effective loaded radius of the assembly and $\lambda$ the reflector savings. Through Equation (10) a measurement of $B_{r}$ can be interpreted as a measurement of $\lambda$. For this purpose it was necessary to establish a way of defining the effective loaded radii of the assemblies, since neither were they exactly cylindrical nor was there a definite boundary between the core and the reflector. The procedure decided on was simply to equate the core cross sectional area to the area of a lattice cell times the number of loaded channels. $R$ was then defined by

$$
\text { Loaded area } \doteq \pi R^{2} \text {. }
$$

The above definition of effective loaded radius has been maintained throughout this work.

\section{Buckling and Reflector Savings}

When both radial and axial flux traverse measurements were made, the reflector savings was determined from the radial flux plots, and the buckling was deduced from the usual expression

$$
B^{2}=B_{r}{ }^{2}-m^{-2} .
$$

Generally six independent measurements of $m$ were made, and the average error and probable error were determined from these. Six independent measurements of $B_{r}{ }^{2}$ were also carried out, and the average and probable error deduced from these. The probable error of $B^{2}$ was found by compounding the errors in $B_{r}{ }^{2}$ and $m^{-2}$ as from random sources.

In some cases it was profitable to find $B^{2}$ and $\lambda$ from axial measurements alone. The procedure then was to measure relaxation lengths $L_{i}$ at many values $\mathcal{N}_{i}$ of rods loaded (hence at many values of loaded radius $R_{i}$ ). It was assumed that $\lambda$ did not depend on the value of $R$, and therefore that

$$
B^{2}=\left(\frac{a}{R_{i}+\lambda}\right)^{2}-\left(\frac{1}{L_{i}}\right)^{2}
$$

for all pairs of values $R_{i}, L_{i}$. Equation (13) can be rewritten as

$$
L_{i}=\left[\left(\frac{a}{R_{i}+\lambda}\right)^{2}-B^{2}\right]^{-1 / 2} .
$$

Least-squares fits of the observed $L_{i}$ to this expression led to values of $\lambda$ and $B^{2}$.

This second procedure for obtaining buckling and reflector savings had to be tested for validity, the most likely source of error having been the assumption of constant $\lambda$. These checks were performed:

1) All such measurements were based on 22 pairs of $L_{i}, R_{i}$. The 11 with the smallest $R_{i}$ and the 11 with the largest $R_{i}$ were subjected separately to least-squares fits for $B^{2}$ and $\lambda$, and the results were compared with those found from fitting all the data at once. There was no tendency for either 11 -point set to give systematically a larger or smaller $B^{2}$ or $\lambda$.

2) The buckling and reflector savings of one lattice (1.5:1 volume ratio, $1.15 \%$ enriched uranium) were measured by both methods. The results were in agreement to better than the mutual probable errors ( $\approx 2 \%$ in critical mass). 
3) The critical mass of one lattice (2:1 volume ratio, $1.15 \%$ enriched uranium) was determined from a subcritical approach. The result agreed with that deduced from a 22-axial measurement to within $0.9 \%$.

4) The $1.3 \%$ and $1.15 \%$ enriched uranium lattices were loaded to critical size at WAPD. ${ }^{5}$ The observed critical masses agreed within the mutual probable errors of the experiments with those found by both kinds of Brookhaven buckling measurements.

The buckling and reflector savings of the clean (unpoisoned) lattices are given in Table 3. Figures 12 and 13 are plots of these data against volume ratio.

Table 4 contains bucklings and reflector savings of lattices poisoned with $\mathrm{B}_{2} \mathrm{O}_{3}$.

\section{Intracell Flux Variation and Thermal Utilization}

The observed behavior of the thermal neutron flux within a lattice cell is typified by the results plotted in Figure 14. Two curves of flux distribution are shown; these correspond to the principal directions in the moderator.

From the measured curves, averages of thermal neutron flux over the fuel and moderator and the aluminum cladding were calculated. The results are given in Table 5.

\section{Table 3}

Buckling and Reflector Savings (Clean Lattices)

\begin{tabular}{cccc}
\hline Volume water & & & \\
Volume uranium & Enrichment & $B^{2}\left(\mathrm{~cm}^{-2} \times 10^{4}\right)$ & $\lambda(\mathrm{cm})$ \\
\hline 1 & 1.3 & $32.11 \pm 0.54$ & $7.94 \pm 0.10$ \\
1.5 & & $51.87 \pm 0.50$ & $7.44 \pm 0.10$ \\
2 & & $61.08 \pm 0.32$ & $7.04 \pm 0.06$ \\
3 & & $60.99 \pm 0.26$ & $6.70 \pm 0.05$ \\
4 & & $50.38 \pm 0.27$ & $6.64 \pm 0.07$ \\
1 & 1.15 & $21.33 \pm 0.41$ & $8.10 \pm 0.12$ \\
1.5 & & $40.23 \pm 0.30$ & $7.50 \pm 0.08$ \\
2 & & $48.22 \pm 0.31$ & $7.10 \pm 0.08$ \\
3 & & $47.12 \pm 0.33$ & $6.68 \pm 0.10$ \\
4 & & $36.03 \pm 0.16$ & $6.43 \pm 0.06$ \\
1 & 1.0 & $9.90 \pm 0.54$ & $8.76 \pm 0.15$ \\
1.5 & & $29.63 \pm 0.42$ & $7.47 \pm 0.13$ \\
2 & & $36.07 \pm 0.39$ & $7.04 \pm 0.15$ \\
3 & & $33.15 \pm 0.31$ & $6.51 \pm 0.16$ \\
4 & & $20.96 \pm 0.26$ & $6.46 \pm 0.17$
\end{tabular}

The thermal utilizations were calculated from the defining expression

$$
f=\frac{\bar{\phi}_{u} V_{u} \Sigma_{u}}{\bar{\phi}_{u} V_{u} \Sigma_{u}+\bar{\phi}_{m} V_{m} \Sigma_{m}+\bar{\phi}_{a l} V_{a l} \Sigma_{a l}}
$$

where $\bar{\phi}$ is a flux average, $V$ a volume, and $\Sigma$ a macroscopic cross section, and the subscripts $u, m$, al refer respectively to uranium, moderator, and aluminum. The cross sections, atom densities, etc., used in the analysis are given in Table 16. The final values of thermal utilization are listed in

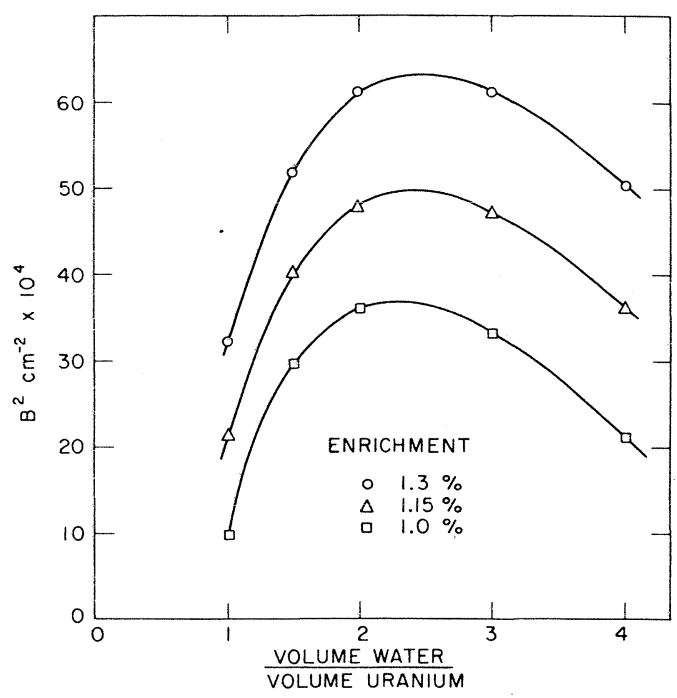

Figure 12. Clean lattice buckling, 0.600 in.

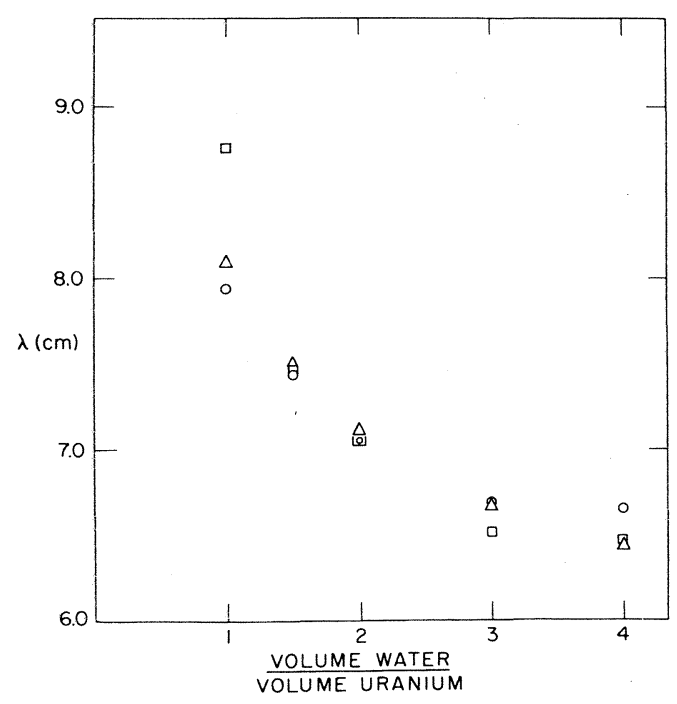

Figure 13. Reflector savings, 0.600 in. 
Table 4

Buckling and Reflector Savings (Poisoned Lattices)

\begin{tabular}{|c|c|c|c|c|}
\hline$\frac{\text { Volume water }}{\text { Volume uranium }}$ & Enrichment & $\mathrm{B}_{2} \mathrm{O}_{3}(\mathrm{mg} / \mathrm{ml})$ & $B^{2}\left(\mathrm{~cm}^{-2} \times 10^{4}\right)$ & $\lambda(\mathrm{cm})$ \\
\hline 1 & 1.3 & 2.587 & $10.67 \pm 1.48$ & $6.32 \pm 0.27$ \\
\hline \multirow[t]{2}{*}{1.5} & & 1.039 & $38.63 \pm 1.13$ & $6.56 \pm 0.26$ \\
\hline & & 3.452 & $10.84 \pm 1.22$ & $5.82 \pm 0.24$ \\
\hline 2 & & 2.587 & $16.38 \pm 0.34$ & $5.90 \pm 0.10$ \\
\hline 3 & & 1.724 & $12.87 \pm 0.83$ & $6.08 \pm 0.12$ \\
\hline \multirow[t]{3}{*}{4} & & 0.500 & $33.21 \pm 0.81$ & $5.90 \pm 0.16$ \\
\hline & & 0.855 & $16.93 \pm 1.00$ & $6.65 \pm 0.25$ \\
\hline & & 1.059 & $10.97 \pm 1.30$ & $6.31 \pm 0.25$ \\
\hline \multirow[t]{2}{*}{1} & 1.15 & 2.98 & $-2.54 \pm 0.58$ & $6.16 \pm 0.15$ \\
\hline & & 3.327 & $-6.44 \pm 0.46$ & $6.23 \pm 0.10$ \\
\hline \multirow[t]{2}{*}{1.5} & & 2.022 & $13.17 \pm 0.43$ & $6.47 \pm 0.10$ \\
\hline & & 3.926 & $-8.18 \pm 0.40$ & $5.76 \pm 0.09$ \\
\hline \multirow[t]{2}{*}{2} & & 1.911 & $12.36 \pm 0.62$ & $6.52 \pm 0.17$ \\
\hline & & 3.00 & $-5.96 \pm 1.00$ & $5.95 \pm 0.10$ \\
\hline 3 & & 1.308 & $7.70 \pm 0.58$ & $6.22 \pm 0.18$ \\
\hline \multirow[t]{3}{*}{4} & & 0.344 & $22.16 \pm 0.36$ & $6.36 \pm 0.16$ \\
\hline & & 0.746 & $5.58 \pm 0.48$ & $6.78 \pm 0.24$ \\
\hline & & 1.300 & $-13.57 \pm 0.46$ & $6.53 \pm 0.21$ \\
\hline
\end{tabular}

Table 6, and are plotted in Figure 15 against volume ratio for the three fuel enrichments.

The results of these measurements are considerably different from those reported at Geneva. The source of the change is to be found in altered technique; the foil holders used in the water previously consisted of aluminum, and a change was made to Lucite. It was established by a series of experiments performed with varying thicknesses of both materials that even the thinnest feasible aluminum foil holders depressed the flux in the moderator appreciably, whereas the perturbation introduced by $1 / 32$ in. of Lucite could be ignored.

Dysprosium detectors have one feature not mentioned earlier. The absorption cross section is not $1 / v$, but drops off faster than this rate with increasing neutron energy. It has been established, ${ }^{2}$ however, that the low-lying resonances of dysprosium shown in the cross section curves of BNL 325 are associated with capture in isotopes other than $D y^{164}$. Therefore the $f$-factor for thermal absorption in $\mathrm{Dy}^{164}$, leading to the 2.32 -hr $\beta$-activity used here, is probably near unity. The effect of any non- $1 / v$ absorption by dysprosium is considered in Appendix II. This appendix also treats the effect of a difference in neutron temperatures between fuel and moderator. The analysis implies that both effects can be ignored.

\section{E. Epi-Cadmium Uranium-239 Production}

Table 7 lists the values of $R_{u}$ and $S$ defined in section III-D. These results were obtained by the straightforward reduction of the data described in that section. For each sample the total number of counts (corrected for background, etc.) accumulated in some arbitrary time was chosen as a measure of the $\mathrm{U}^{239}$ production. This number was divided by any one of the three quantities described in section III-D which measured the quantity of $\mathrm{U}^{238}$ in the sample. The resulting ratio from the bare samples was divided by the similar ratio from the Cd-covered samples to obtain $R_{u}$. Thus each run resulted in three values of $R_{u}$ corresponding to the three assays. Several runs were done on each lattice, so that in all there resulted about nine values of $R_{u}$, which were then averaged to get the final values listed in Table 7 . The irradiation and separation histories of all the samples in a given run were of course identical; the counting histories were different insofar as the samples were rotated among several counters in accordance with the standard $\beta$-counting procedure. The necessary 


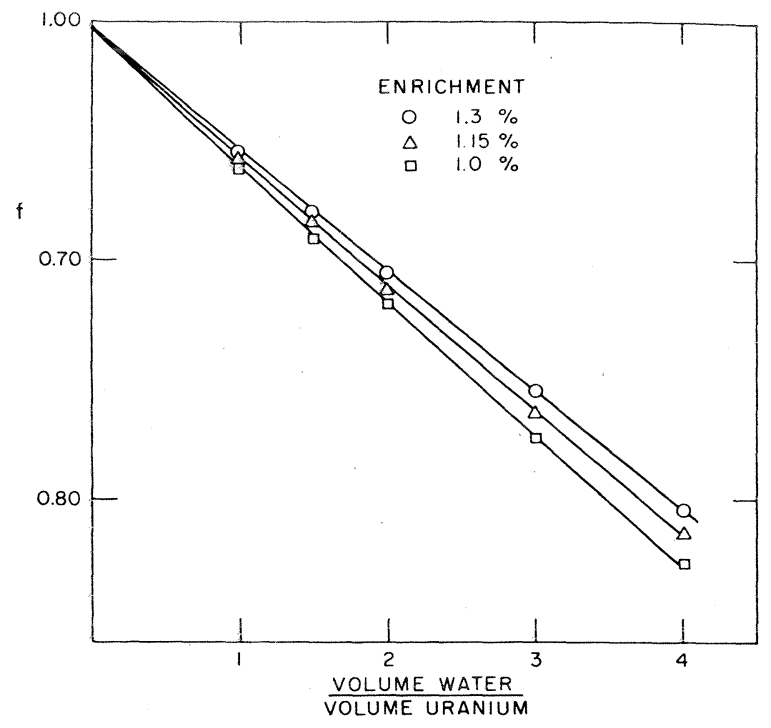

Figure 14. Intracell flux plot, 0.600 in.

Figure 15. Thermal utilization, 0.600 in.

Table 5

Intracell Flux Averages

\begin{tabular}{lcccc}
\hline $\begin{array}{c}\text { Volume water } \\
\text { Volume uranium }\end{array}$ & $\begin{array}{c}\text { Enrichment } \\
\left(\% \mathrm{U}^{235}\right)\end{array}$ & $\bar{\phi}_{m}$ & $\bar{\phi}_{u}$ & $\bar{\phi}_{a l}$ \\
\hline 1 & 1.3 & 1.446 & 1.125 & 1.258 \\
1.5 & & 1.536 & 1.134 & 1.270 \\
2 & & 1.618 & 1.134 & 1.260 \\
3 & & 1.684 & 1.136 & 1.265 \\
4 & & 1.810 & 1.144 & 1.295 \\
1 & 1.15 & 1.421 & 1.119 & 1.245 \\
1.5 & & 1.449 & 1.124 & 1.250 \\
2 & & 1.591 & 1.150 & 1.303 \\
3 & & 1.632 & 1.126 & 1.255 \\
4 & & 1.722 & 1.137 & 1.283 \\
1 & 1.0 & 1.410 & 1.103 & 1.220 \\
1.5 & & 1.478 & 1.119 & 1.243 \\
2 & & 1.506 & 1.111 & 1.230 \\
3 & & 1.614 & 1.115 & 1.230 \\
4 & & 1.706 & 1.123 & 1.252 \\
\hline
\end{tabular}

Table 6

Thermal Utilization

\begin{tabular}{lccc}
\hline & \multicolumn{3}{c}{ Enrichment } \\
\cline { 2 - 4 } $\begin{array}{c}\text { Volume } \\
\text { ratio }\end{array}$ & 1.3 & 1.15 & 1.0 \\
\hline 1 & 0.946 & 0.942 & 0.937 \\
1.5 & 0.920 & 0.917 & 0.909 \\
2 & 0.893 & 0.887 & 0.881 \\
3 & 0.845 & 0.836 & 0.825 \\
4 & 0.795 & 0.787 & 0.773 \\
\hline
\end{tabular}

Table 7

Epi-Cd U ${ }^{239}$ Fraction

\begin{tabular}{ccc}
\hline$V_{\mathrm{H}_{2} \mathrm{O}} / V_{u}$ & $R_{u}=$ Cd ratio & $S=\frac{\text { epi-Cd }}{\text { sub-Cd }}=\frac{1}{R_{u}-1}$ \\
\hline 1 & $1.50 \pm 0.05$ & $2.00 \pm 0.20$ \\
2 & $1.99 \pm 0.02$ & $1.01 \pm 0.02$ \\
3 & $2.26 \pm 0.03$ & $0.79 \pm 0.02$ \\
4 & $2.66 \pm 0.13$ & $0.60 \pm 0.02$ \\
\hline
\end{tabular}

Table 8

Gold Cadmium Ratios

\begin{tabular}{cc}
\hline$V_{\mathrm{H}_{2} \mathrm{O}} / V_{u}$ & $R_{0}$ \\
\hline 1 & 1.18 \\
1.5 & 1.24 \\
2 & 1.32 \\
3 & 1.47 \\
4 & 1.55 \\
\hline
\end{tabular}

Table 9

$\mathrm{U}^{238}$ Fission Fractions

\begin{tabular}{crrr}
\hline$\frac{\text { Volume water }}{\text { Volume uranium }}$ & $F(1.0 \%)$ & $F(1.15 \%)$ & $F(1.3 \%)$ \\
\hline 1 & 0.1918 & 0.1784 & 0.1780 \\
1.5 & .1461 & .1370 & .1346 \\
2 & .1202 & .1094 & .1100 \\
3 & .0914 & .0816 & .0871 \\
4 & .0708 & .0739 & .0734 \\
\hline
\end{tabular}


intercalibration factors between the counters were obtained by correcting each count to some early time by means of the decay curve of Figure 6.

\section{F. Gold Cadmium Ratio in Fuel}

Routine reduction of the counting data resulted in the values of $R_{0}$ (defined in section III-E) listed in Table 8.

\section{G. Uranium-238 Fission Fraction}

The reduction of the data has already been described in section III-F. Table 9 lists the values of $F$ thus obtained. Each value is the average of several runs.

\section{H. Epi-Cadmium Uranium-235 Fission Rate}

The reduction of these data is similar to that described in section III-F. It is necessary, however, to subtract from each catcher foil activity the contribution due to $\mathrm{U}^{238}$ fissions. The results of the fast fission fraction measurements permit this correction to be made, and the results are given in Table 10. Unfortunately the correction is not small; the fraction of the epi-Cd fission product activity attributable to $\mathrm{U}^{238}$ fission was generally of the order of 70 to $90 \%$. The uncertainty in the epi-Cd $\mathrm{U}^{235}$ fission fraction is then quite large, of course. Nevertheless, the results of independent runs generally were in fair agreement - although some deviations from the mean were as high as $20 \%$, most were less than $10 \%$. Statistical counting errors were normally much lower. There is reason to believe, from comparison of these results with similar ones obtained at WAPD ${ }^{6}$ and from comparison with $\mathrm{Au} \mathrm{Cd}$ ratios listed in Table 8 (see section $\mathrm{V}-\mathrm{E}$ ), that many of the values of $\rho_{m}$ listed in Table 10 are too low, some perhaps by as much as a factor of 2 .

Table 10

Epi-Cd Fission Fractions in $\mathrm{U}^{235}$

\begin{tabular}{lrrr}
\hline$V_{\mathrm{H}_{2} \mathrm{O}} / V_{u}$ & $\rho_{m}(1 \%)$ & $\rho_{m}(1.15 \%)$ & $\rho_{m}(1.3 \%)$ \\
\hline 1 & 0.144 & 0.174 & 0.207 \\
1.5 & .116 & .105 & .155 \\
2 & .078 & .082 & .117 \\
3 & .045 & .054 & .062 \\
4 & .032 & .040 & .048 \\
\hline
\end{tabular}

\section{Boron-Cadmium Danger Coefficients}

This experiment and its results were described in an earlier report, ${ }^{3}$ and are included here primarily for completeness.

When an assembly is sufficiently near to being critical, the reciprocal of the count rate observed with an appropriate detector is linear in $k_{\text {eff }}$ :

$$
1 / R \propto 1-k_{\mathrm{eff}}=1-k_{\infty} \exp \left(-M^{2} B_{g}^{2}\right) .
$$

(It should be observed that, although a particular form of the non-leakage probability has been chosen, the results of the measurement do not depend on this selection.) For sufficiently small increments, the change in $k_{\text {ef } f}$ caused by adding a uniform poison is proportional to the macroscopic cross section of the poison:

$$
\delta k_{\text {eff }} \propto \Sigma_{\text {poison }} .
$$

The subscripts 0,1 , and 2 are respectively used to refer to the situations when the moderator water was clean, when it contained boron, and when it contained boron and cadmium. The changes in $k_{\text {ef } \mathrm{f}}$ induced by adding boron and cadmium are denoted respectively as $-\delta$ and $-\Delta$. Then

$$
\begin{aligned}
& 1 / R_{0} \propto 1-k_{0}=1-k_{1}-\delta, \\
& 1 / R_{1} \propto 1-k_{1}, \\
& 1 / R_{2} \propto 1-k_{2}=1-k_{1}+\Delta, \\
& \left(1 / R_{1}\right)-\left(1 / R_{0}\right) \propto \delta \propto \Sigma_{B}, \\
& \left(1 / R_{2}\right)-\left(1 / R_{1}\right) \propto \Delta \propto \Sigma_{C d} .
\end{aligned}
$$

Therefore

$$
\frac{\left(1 / R_{2}\right)-\left(1 / R_{1}\right)}{\left(1 / R_{1}\right)-\left(1 / R_{0}\right)}=\frac{\Sigma_{C d}}{\Sigma_{B}} .
$$

The macroscopic cross sections are proportional to the molar fractions $M$ times the microscopic cross sections. Thus

$$
\frac{\left(1 / R_{2}\right)-\left(1 / R_{1}\right)}{\left(1 / R_{1}\right)-\left(1 / R_{0}\right)}=\frac{M_{C d} \sigma_{C d}}{M_{B} \sigma_{B}} .
$$

The cross sections which should be used in the analysis are averages over the energy spectrum in the reactor. In practice the averages were taken over a Maxwell distribution as functions of the characteristic energy $k T$. Both numerical and analytic integrations were performed as checks. It is realized that it would have been more suitable to use a Maxwellian energy distribution with a $1 / E$ tail. This has not been done, and so the size 
of the error introduced by the approximation has not been found.

The result of comparing the measured values of danger coefficients led to the result that in the one assembly studied (3:1 volume ratio, $1.15 \%$ rods),

$$
\sigma_{C d} / \sigma_{B}=4.73 \pm 0.17 \text {. }
$$

These results are fitted by a neutron temperature of $T=304^{\circ} \pm 16^{\circ} \mathrm{K}$. The moderator water temperature was actually $297^{\circ} \mathrm{K}$.

\section{J. Two-Region Loading ${ }^{4}$}

The two-region lattice results were analyzed with the aid of two-group, two-region theory. Critical masses were found from extrapolation of the multiplication curves in two cases: the entire lattice containing $1.15 \%$ rods, and the inner 19 rods being $1.0 \%$ enriched. It was assumed that the buckling in the $1.15 \%$ enriched region was known from exponential experiments. Knowledge of this buckling and of the two critical sizes was then sufficient information from which to deduce the buckling in the $1.0 \%$ region.

The result obtained was

$$
B^{2}=(33.36 \pm 0.50) \times 10^{-4} \mathrm{~cm}^{-2}
$$

compared with that from exponential experiments

$$
B^{2}=(32.93 \pm 0.34) \times 10^{-4} \mathrm{~cm}^{-2}
$$

The validity of the analysis and the method thus seems to be established.

However, two features of a measurement of this sort require close scrutiny. The first is the problem of the definition of the boundary between the inner and outer regions, which is largely arbitrary. In the analysis above it was assumed that the inner region was a cylinder of the same total area as the lattice cells it contained. When there are so few rods in the central region, the results are sensitive to this assumption.

Second, in order to make the analysis tractable, the fast diffusion coefficients of the two regions were assumed to be equal. The assumption was certainly correct in this case, but there are clearly limitations on general applicability.

\section{INTERPRETATION}

\section{A. Conventional Four-Factor Formula}

In section IV measured values are tabulated of bucklings (and reflector savings), intracell flux var-
Table 11

Fast Fission Factor

\begin{tabular}{lcccc}
\hline & \multicolumn{3}{c}{ Enrichment } & \\
\cline { 2 - 4 } $\begin{array}{c}\text { Volume } \\
\text { ratio }\end{array}$ & 1.3 & 1.15 & 1.0 & Average \\
\hline 1 & 1.099 & 1.098 & 1.105 & 1.101 \\
1.5 & 1.075 & 1.076 & 1.081 & 1.077 \\
2 & 1.063 & 1.063 & 1.066 & 1.064 \\
3 & 1.049 & 1.045 & 1.051 & 1.048 \\
4 & 1.042 & 1.041 & 1.040 & 1.041 \\
\hline
\end{tabular}

iation, epi-Cd/sub-Cd U ${ }^{239}$ production, and fast fission fractions in $\mathrm{U}^{238}$ and $\mathrm{U}^{235}$. Some of these quantities have already been interpreted in terms of the four-factor formula - in particular thermal utilization, which is listed in Table 6.

The fast effect, $\varepsilon$, is given in terms of the measured quantities by the familiar expression

$$
\varepsilon=1+\frac{F}{\nu_{25}}\left(\nu_{28}-1-\alpha_{28}\right) .
$$

It was assumed that $\nu_{28}=2.5$, essentially because no better value exists. The value of $\alpha_{28}$ was determined by numerical integration of the appropriate cross sections in $\mathrm{U}^{238}$ over the fast region. The value so obtained was 0.107 , but the values of $\varepsilon$ (and $p$ ) should not be very much changed by an improvement in the value of $\alpha_{28}$. The value of $\nu_{25}$ was taken also to be 2.50 ; the more recent

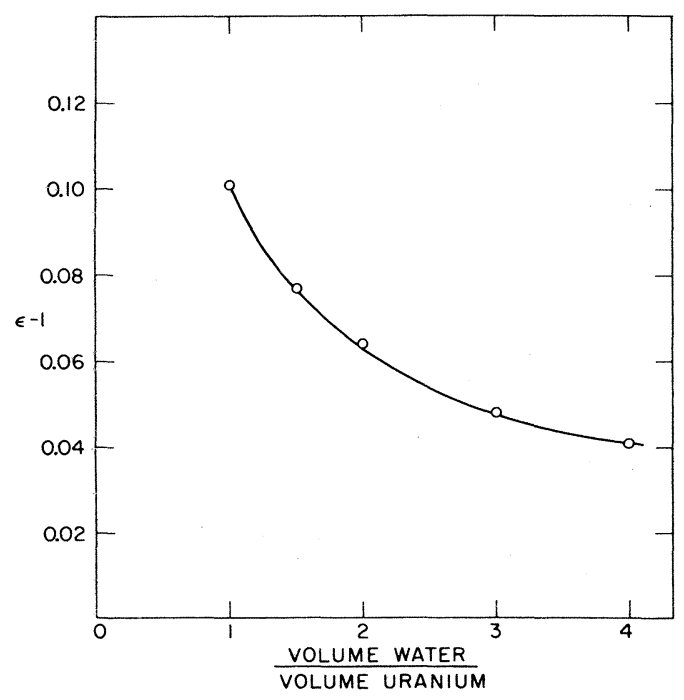

Figure 16. Fast effect, 0.600 in. 
Table 12

Resonance Escape Probability

\begin{tabular}{cc}
\hline Volume ratio & \\
\hline 1 & $0.720 \pm 0.027$ \\
2 & $0.841 \pm 0.004$ \\
3 & $0.868 \pm 0.003$ \\
4 & $0.909 \pm 0.008$ \\
\hline
\end{tabular}

"best" value of 2.46 would raise the final values of $\varepsilon$ by about $0.1 \%$. The major systematic uncertainty in $\varepsilon$, however, is the value of $\nu_{28}$. Table 11 lists the values of $\varepsilon$ for the 0.600 -in. lattices. The averages are plotted in Figure 16.

The resonance escape probability, $p$, is given by the following expression, which is derived in Appendix $\mathrm{V}$ from considerations of the neutron cycle:

$$
p=\frac{1+\left[(\varepsilon-1) \alpha_{28} / \varepsilon\left(\nu_{28}-1-\alpha_{28}\right) \mathcal{L}_{1}\right]}{1+\left[f \Sigma \mathcal{L}_{2} \mathcal{L}_{s} S(1-\delta / S) /(1+\delta)\right]} .
$$

Here $\mathcal{L}_{1}, \mathcal{L}_{2}$, and $\mathcal{L}_{s}$ are non-leakage probabilities in the fast, resonance, and thermal regions respectively, $\Sigma=\sum_{28}^{\mathrm{abs}} / \sum_{\mathrm{fuel}}^{\mathrm{abs}}$, and $\delta$ is defined by the fact that $\delta / S$ is the fraction of epi-Cd capture which is $1 / v$ capture. Thus if $S=$ epi-Cd/sub-Cd capture in $\mathrm{U}^{238}$,

$$
S \frac{(1-\delta / S)}{(1+\delta)}=\frac{\text { non }-1 / v \text { capture in } \mathrm{U}^{238}}{1 / v \text { capture in } \mathrm{U}^{238}} .
$$

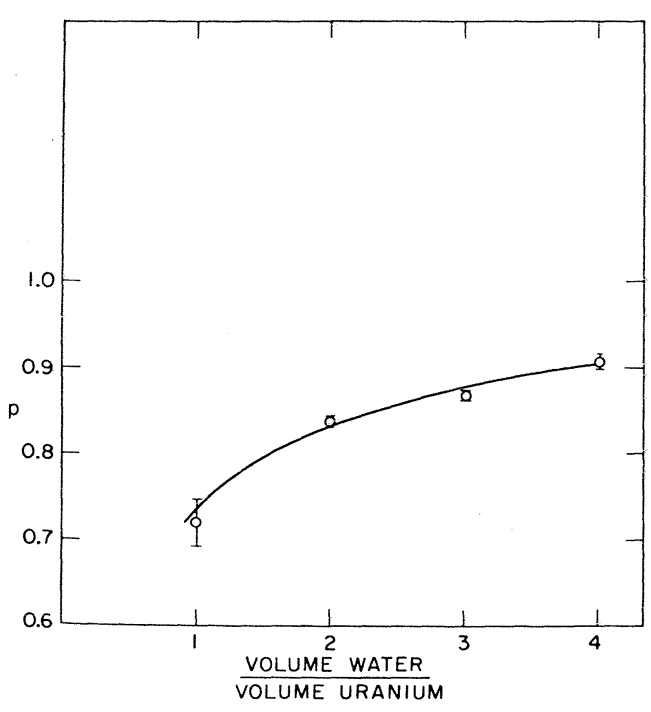

Figure 17. Resonance escape probability, $0.600 \mathrm{in.}$
One may determine $\delta$ either from the quantity $\rho_{m}$ described above (sections III-G and IV-H) or in terms of the Cd ratio of thin Au foils irradiated in the fuel rods. Appendix III derives these relationships. The values of $p$ obtained are listed in Table 12 and are plotted in Figure 17.

The terms involving $\delta$ are included in order to avoid counting epi-Gd $1 / v$ absorption in $\mathrm{U}^{238}$ twice in the neutron cycle, namely in $f$ and in $p$. The decision to put epi-Cd $1 / v$ absorption in $\mathrm{U}^{238}$ in $f$ rather than in $p$ is arbitrary; had it been included in $p$, the $\delta$-terms would be absent, but an analogous correction term would have been necessary in the expression for $f$. All $1 / v$ processes have thus been called "thermal" (in addition to sub-Cd processes that are non- $1 / v$; although the departure of these from $1 / v$ behavior is generally negligible or is corrected for by use of the $f$-factor). In line with this choice, it seemed more natural to include epi-Cd $1 / v$ capture in $f$ (thermal utilization) rather than in $p$ (resonance escape probability).

The non-leakage terms $\mathcal{L}_{1}, \mathcal{L}_{2}, \mathcal{L}_{s}$ are estimated from the measured bucklings and reasonable guesses of the fast and resonance ages. The values of $p$ are not sensitive to these guesses. In section $\mathrm{V}$-D are presented expressions for $\varepsilon$ and $p$ which include effects of epi-thermal fission in $\mathrm{U}^{235}$, derived from a group-diffusion point of view using three groups. The resulting expressions in the limit of no epi-thermal $\mathrm{U}^{235}$ fission are very slightly different from those used in this section; the differences are in the effects of the non-leakage probabilities and are therefore quite small.

\section{B. Migration Area}

Before a discussion of the way in which the migration area was determined from these experiments, it is necessary to comment on the extent to which the concept is well defined. This must be done because $M^{2}$ is a derived quantity, not having been measured directly. Therefore the values it takes on depend on the features of the derivation, and these contain implicit assumptions about such things as the nature of the neutron slowing-down process.

Most commonly, $M^{2}$ has the meaning suggested by its name:

$$
M^{2}=\bar{r}^{2} / 6,
$$

$\bar{r}^{2}$ being the second moment of the capture distribution about a point source in an infinite region 
of moderator. The importance of the concept in reactor theory is found in the more elementary treatments, in which the expressions for the neutron non-leakage probability $\mathcal{L}$ contain $M^{2}$. There is for example good theoretical justification in the case of the one-region homogeneous overmoderated graphite-enriched uranium system for the relation

$$
\mathcal{L}=\left(1+L^{2} B_{g}{ }^{2}\right)^{-1} \exp \left(-\tau B_{g}{ }^{2}\right)
$$

with

$$
M^{2}=L^{2}+\tau .
$$

The derivation of Equation (19) depends however on the description of slowing down as continuous in the energy variable. Whereas this assumption is not very bad when the moderator is carbon, it is questionable for reactors containing deuterium and is drastic for those with hydrogen. Nevertheless, such expressions for non-leakage are so useful that they are still used even where rigorous theoretical justification is lacking. For instance, Equation (19) is usually used in discussions of the kinetics of heavy water moderated reactors, even though the basis for doing so is only approximate. In practice, the use of this scheme to calculate reactivities of heavy water reactors with $k_{\infty}$ substantially greater than unity may lead to errors of several percent. Accuracy of this amount is however sufficient for most purposes.

The situation for light water moderated reactors is not this good. There are no simplified schemes for slowing down in hydrogenous materials, and hence there is no clear theoretical basis for choice of a form for the non-leakage probability. There are certain experimental indications; the capture distribution in light water can be matched with reasonable accuracy by a sum of three exponentials, and this implies that it should be possible to describe leakage through a three-group diffusion formalism. This is however by no means a settled matter.

Since the usefulness for reactors of the migration area is associated with its role in determining the neutron leakage probabilities, and since for wateruranium systems the connection with the slowingdown and diffusion areas is not direct, it is best to derive experimental values from the behavior of the non-leakage itself. To do so is equivalent to basing the measurement on the critical equation.

As has been pointed out, however, it is not clear how the critical equation for these systems should be chosen. Therefore several possible forms have been selected from elementary reactor theory, and the analysis of the measurements has been carried out with the use of each. This course has served to indicate how the measured values depend on the degree of arbitrariness in the theory, and to point out which features of the results are independent of the resolution of this uncertainty. The critical equations which have been tried are taken from one-group, two-group, three-group, age-diffusion, and age theories. The last two differ in that the first contains the assumption of a thermal group and the second does not. The separation of diffusion areas among several groups in a multi-group model is somewhat arbitrary; three such divisions have been tried in connection with the three-group model. The seven critical equations used in the analysis are given in Table 13.

This analysis of the experiments has rested on a four-factor interpretation of the neutron cycle:

$$
k_{\infty}=\varepsilon p f \eta=F\left(B^{2}\right),
$$

$F\left(B^{2}\right)$ being the functional for $m$ in the critical equation. The experimental procedures have been described in earlier reports. They depend in essence on the point that if $f, \eta$, and $B^{2}$ are varied simultaneously through a single means, the function

$$
F\left(B^{2}\right) / f \eta
$$

is a constant. $F$ however contains $M^{2}$ or some part thereof as a parameter. The value of this parameter is then so chosen as to cause the expression (22) to have the least variation.

Two methods have been used to change $f, \eta$, and $B^{2}$. In the first, a boric acid solution was added to the moderator water. This altered only

\section{Table 13}

Critical Equations Used

\begin{tabular}{ll}
\hline One-group & $k_{\infty}=1+M^{2} B^{2}$ \\
Two-group & $k_{\infty}=\left(1+L_{s}{ }^{2} B^{2}\right)\left(1+L_{f}{ }^{2} B^{2}\right)$ \\
Three-group a) & $k_{\infty}=\left(1+L_{s}{ }^{2} B^{2}\right)\left(1+5 B^{2}\right)\left(1+L_{2}{ }^{2} B^{2}\right)$ \\
b) & $k_{\infty}=\left(1+L_{s}{ }^{2} B^{2}\right)\left(1+7.5 B^{2}\right)\left(1+L_{2}{ }^{2} B^{2}\right)$ \\
c) & $k_{\infty}=\left(1+L_{s}{ }^{2} B^{2}\right)\left(1+10 B^{2}\right)\left(1+L_{2}{ }^{2} B^{2}\right)$ \\
Age & $k_{\infty}=\exp \left(M^{2} B^{2}\right)$ \\
Age-diffusion & $k_{\infty}=\left(1+L_{s}{ }^{2} B^{2}\right) \exp \left(\tau B^{2}\right)$ \\
\hline
\end{tabular}


$f$ and $B^{2} ; \eta$ remained unchanged (as did $\varepsilon$ and $p$ ). At each level of boron poisoning, $B^{2}$ and $f$ were determined.

The second procedure involved combining information obtained with the three sets of uranium rods. Lattices with identical volume ratios but containing fuel of different enrichments differed only in their values of $B^{2}, f$, and $\eta$. The first two were measured, and values of $\eta$ were assigned in a manner discussed below.

The experimental methods by which the buckling was measured have been discussed earlier in this report. In all, 33 measurements of $B^{2}$ were made, 15 with unpoisoned and 18 with poisoned lattices, with the results listed in Tables 3 and 4 .

The method of intracell flux traverses by which thermal utilization was measured has also been described earlier. It was not necessary however to determine the thermal flux variation separately for lattices which differed only in boron poisoning, since it was observed that the amount of boron involved did not change this distribution noticeably. Therefore intracell flux distributions were measured only when the lattices contained no poison. The boron content in the water at other times was measured by the analytical group of the Chemistry Department, and $f$ was determined according to the prescription of Equation (15), the unpoisoned lattice flux averages being used. The cross sections used in the calculation were taken from BNL 325; all were averaged over a Maxwell distribution with most probable speed $2200 \mathrm{~m} / \mathrm{sec}$.

Since $\eta$ was not measured, it was necessary to choose a consistent method whereby it could be calculated. The scheme selected is contained in the expression

$$
\eta\left(1+\frac{n_{28} \sigma_{a}{ }^{28}}{n_{25} \sigma_{a}{ }^{25}}\right)=\text { constant } .
$$

The value of the constant was first determined from insertion of $\eta=1.310, n_{28} / n_{25}=137.8$ (values

Table 14

Values of $\eta$ Used in Analysis for $M^{2}$

\begin{tabular}{cc}
\hline Enrichment & $\eta$ (based on $\left.\eta_{\text {nat }}=1.310\right)$ \\
\hline $1.3 \%$ & 1.5674 \\
$1.15 \%$ & 1.5182 \\
$1.0 \%$ & 1.4745 \\
\hline
\end{tabular}

Table 15

Quantities Used in $M^{2}$ Analysis

\begin{tabular}{|c|c|c|c|c|}
\hline $\begin{array}{l}\text { Volume } \\
\text { ratio }\end{array}$ & $\begin{array}{c}\text { Enrichment } \\
\left(\% \mathrm{U}^{235}\right)\end{array}$ & $\begin{array}{c}\mathrm{B}_{2} \mathrm{O}_{3} \\
(\mathrm{mg} / \mathrm{ml})\end{array}$ & $\begin{array}{c}L^{2} \\
\left(\mathrm{~cm}^{2}\right)\end{array}$ & $f$ \\
\hline \multirow[t]{6}{*}{1} & 1.3 & 0 & 2.205 & 0.9462 \\
\hline & 1.3 & 2.587 & 2.062 & .8808 \\
\hline & 1.15 & 0 & 2.403 & .9421 \\
\hline & 1.15 & 2.98 & 2.200 & .8627 \\
\hline & 1.15 & 2.327 & 2.179 & .8543 \\
\hline & 1.0 & 0 & 2.592 & .9370 \\
\hline \multirow[t]{7}{*}{1.5} & 1.3 & 0 & 2.382 & .9203 \\
\hline & 1.3 & 1.039 & 2.277 & .8800 \\
\hline & 1.3 & 3.452 & 2.068 & .7989 \\
\hline & 1.15 & 0 & 2.585 & .9159 \\
\hline & 1.15 & 2.022 & 2.354 & .8373 \\
\hline & 1.15 & 3.926 & 2.179 & .7745 \\
\hline & 1.0 & 0 & 2.778 & .9086 \\
\hline \multirow[t]{6}{*}{2} & 1.3 & 0 & 2.566 & .8933 \\
\hline & 1.3 & 2.587 & 2.221 & .7730 \\
\hline & 1.15 & 0 & 2.767 & .3872 \\
\hline & 1.15 & 1.911 & 2.454 & .7874 \\
\hline & 1.15 & 3.00 & 2.330 & .7454 \\
\hline & 1.0 & 0 & 2.959 & .8812 \\
\hline \multirow[t]{5}{*}{3} & 1.3 & 0 & 2.895 & .8453 \\
\hline & 1.3 & 1.724 & 2.513 & .7333 \\
\hline & 1.15 & 0 & 3.109 & .8360 \\
\hline & 1.15 & 1.308 & 2.790 & .7446 \\
\hline & 1.0 & 0 & 3.324 & .8246 \\
\hline \multirow[t]{9}{*}{4} & 1.3 & 0 & 3.240 & .7949 \\
\hline & 1.3 & 0.500 & 3.061 & .7504 \\
\hline & 1.3 & 0.855 & 2.995 & .7218 \\
\hline & 1.3 & 1.059 & 2.878 & .7063 \\
\hline & 1.15 & 0 & 3.437 & .7869 \\
\hline & 1.15 & 0.344 & 3.296 & .7550 \\
\hline & 1.15 & 0.746 & 3.145 & .7207 \\
\hline & 1.15 & 1.300 & 2.961 & .6783 \\
\hline & 1.0 & 0 & 3.661 & .7727 \\
\hline
\end{tabular}

for natural uranium) and Maxwell-averaged $\sigma_{a}$ 's from BNL 325. Then $\eta$ at the three enrichments used in the experiment could be calculated. The values resulting from the analysis are given in Table 14

The choice $\eta=1.310$ for natural uranium deserves comment here. The old Manhattan Project value for this parameter was 1.315 . Recent cross section measurements indicate a number in the neighborhood of $1.330 \pm 0.020$. However, most recent criticality measurements (including these; see section $\mathrm{V}-\mathrm{C}$ ) imply a value around 1.310 . The most complete collection of Brookhaven data leads 
Table 16

\begin{tabular}{|c|c|c|c|c|c|}
\hline \multicolumn{6}{|c|}{ Constants Used in $M^{2}$ Analysis } \\
\hline & Atoms $/ \mathrm{cm}^{3}$ & $\begin{array}{c}\sigma_{a}(2200 \mathrm{~m} / \mathrm{s}) \\
\text { (barns) }\end{array}$ & $\begin{array}{c}\sigma_{a}(\text { Maxwell }) \\
(\text { barns })\end{array}$ & $\begin{array}{c}\sum_{a}(\text { Maxwell }) \\
\left(\mathrm{cm}^{-1}\right)\end{array}$ & $\begin{array}{r}L_{2}{ }^{2} \\
\left(\mathrm{~cm}^{2}\right)\end{array}$ \\
\hline $\mathrm{H}$ (in water) & $6.689 \times 10^{22}$ & 0.330 & 0.2926 & 0.01957 & \\
\hline $\mathrm{Al}$ & $6.031 \times 10^{22}$ & 0.230 & 0.2039 & .01230 & \\
\hline $\mathrm{U}^{235}$ & & 687 & 597.5 & & \\
\hline $\mathrm{U}^{238}$ & & 2.75 & 2.438 & & \\
\hline $\mathrm{B}$ & & 760 & & & \\
\hline $\mathrm{U}^{235}(1 \% \mathrm{U})$ & $4.971 \times 10^{20}$ & & & .2970 & \\
\hline $\mathrm{U}^{238}(1 \% \mathrm{U})$ & $4.732 \times 10^{22}$ & & & .1154 & \\
\hline $\mathrm{U}^{235}(1.15 \%)$ & $5.540 \times 10^{20}$ & & & .3310 & \\
\hline $\mathrm{U}^{238}(1.15 \%)$ & $4.731 \times 10^{22}$ & & & .1153 & \\
\hline $\mathrm{U}^{235}(1.3 \%)$ & $6.288 \times 10^{20}$ & & & .3757 & \\
\hline $\mathrm{U}^{238}(1.3 \%)$ & $4.719 \times 10^{22}$ & & & .1151 & \\
\hline $\mathrm{H}_{2} \mathrm{O}$ & & & & & 7.56 \\
\hline $1 \% \mathrm{U}$ & & & & & 1.95 \\
\hline $1.15 \% \mathrm{U}$ & & & & & 1.80 \\
\hline $1.3 \% \mathrm{U}$ & & & & & 1.64 \\
\hline
\end{tabular}

to the result $1.309 \pm 0.006 .^{7}$ This result is much more precise than that deduced from cross sections, and so it has been used here. As pointed out in the Geneva paper on the Brookhaven work on water lattice measurements, ${ }^{1}$ the inferred values of $M^{2}$ do not depend strongly on the assumed $\eta$ for natural uranium. Thus in any case this assumption is not a large source of error.

The analysis used is described as follows.

One-group. The basic expression is

$$
\varepsilon p f \eta=1+M^{2} B^{2} .
$$

Thus $f \eta$ is a linear function of $B^{2}$. A plot of $f \eta$ against $B^{2}$ should be a straight line whose slope is $M^{2}(\varepsilon p)^{-1}$ and whose intercept at $B^{2}=0$ is $(f \eta)_{0}=$ $(\varepsilon p)^{-1}$. The data were fitted by least squares to a straight line, and $M^{2}$ was evaluated.

Two-group. The model is that of a slowing-down group and a thermal group. The thermal diffusion areas are assumed known, and the fast diffusion area remains to be evaluated from the data. The basic relation is

$$
\varepsilon p f \eta=\left(1+L_{s}{ }^{2} B^{2}\right)\left(1+L_{f}{ }^{2} B^{2}\right) .
$$

Thus the quantity

$$
u=\frac{f \eta}{1+L_{s}^{2} B^{2}}
$$

is linearly related to $B^{2}$. A plot of $u$ vs $B^{2}$ should be a straight line whose slope is $L_{f}{ }^{2}$ and whose intercept is $(u)_{0}=(\varepsilon p)^{-1} \cdot M^{2}$ is defined as $M^{2}=$ $L_{s}{ }^{2}+L_{f}{ }^{2}$.

The thermal diffusion areas were calculated with the aid of the usual capture-density weight factors,

$$
L_{s}{ }^{2}=f\left(L_{s}{ }^{2}\right)_{\text {fuel }}+(1-f)\left(L_{s}{ }^{2}\right)_{\text {water }},
$$

and were then corrected for the existence of air void and aluminum clad about the fuel. In this connection, it was assumed that the aluminum acts as a void also. The diffusion lengths so obtained are given in Table 15. The cross sections and necessary geometrical data are listed in Tables 2 and 16.

Once $L_{s}{ }^{2}$ had been obtained, $u$ was calculated, and least-squares fits of $u$ vs $B^{2}$ led to values of $L_{f}{ }^{2}$.

Three-group. The basic equation is

$$
\varepsilon p f \eta=\left(1+L_{s}{ }^{2} B^{2}\right)\left(1+L_{1}{ }^{2} B^{2}\right)\left(1+L_{2}{ }^{2} B^{2}\right) .
$$

It is assumed that $L_{s}{ }^{2}$ and $L_{1}{ }^{2}$ are known. The quantity

$$
v=\frac{f \eta}{\left(1+L_{s}^{2} B^{2}\right)\left(1+L_{1}^{2} B^{2}\right)}
$$

is therefore linearly related to $B^{2}$. The slope of the straight-line plot is $L_{2}{ }^{2}$. The form of the equation makes no distinction between $L_{1}{ }^{2}$ and $L_{2}{ }^{2}$, therefore $L_{1}{ }^{2}$ can be visualized as belonging to either the fast group or the intermediate energy group. 
Table 17

Results of $M^{2}$ Analyses

\begin{tabular}{lcccc}
\hline $\begin{array}{c}\text { Volume } \\
\text { ratio }\end{array}$ & $\begin{array}{c}\text { Enrichment } \\
\text { variation }\end{array}$ & $\begin{array}{c}\text { Poisoned } \\
1.3 \% \text { lattices }\end{array}$ & $\begin{array}{c}\text { Poisoned } \\
1.15 \% \text { lattices }\end{array}$ & $\begin{array}{c}\text { Combined } \\
\text { analysis }\end{array}$ \\
\hline & & One-group, $M^{2}=\left(\mathrm{cm}^{2}\right)$ & \\
1 & 34.20 & 35.95 & 36.87 & $36.85 \pm 0.95$ \\
1.5 & 38.41 & 38.50 & 36.57 & $36.81 \pm 0.89$ \\
2 & 34.98 & 36.92 & 34.80 & $35.45 \pm 0.90$ \\
3 & 36.05 & 33.10 & 31.91 & $32.76 \pm 1.18$ \\
4 & 34.20 & 32.17 & 30.74 & $31.04 \pm 1.17$ \\
\hline
\end{tabular}

Two-group, $L_{f}{ }^{2}=\left(\mathrm{cm}^{2}\right)$

$\begin{array}{lllll}1 & 31.85 & 33.29 & 34.38 & 34.41 \\ 1.5 & 35.89 & 35.48 & 33.76 & 34.00 \\ 2 & 32.36 & 33.96 & 31.68 & 32.27 \\ 3 & 32.88 & 29.41 & 28.23 & 29.12 \\ 4 & 30.59 & 28.26 & 27.25 & 27.48\end{array}$

Three-group $\left(L_{1}^{2}=5 \mathrm{~cm}^{2}\right), L_{2}^{2}=\left(\mathrm{cm}^{2}\right)$

$\begin{array}{lllll}1 & 26.31 & 27.62 & 29.16 & 29.05 \\ 1.5 & 29.63 & 29.55 & 28.31 & 28.38 \\ 2 & 26.00 & 27.86 & 26.07 & 26.51 \\ 3 & 26.54 & 23.52 & 22.60 & 23.32 \\ 4 & 24.14 & 22.54 & 22.02 & 22.07\end{array}$

Three-group $\left(L_{1}{ }^{2}=7.5 \mathrm{~cm}^{2}\right), L_{2}{ }^{2}=\left(\mathrm{cm}^{2}\right)$

$\begin{array}{lllll}1 & 23.54 & 24.99 & 26.53 & 26.42 \\ 1.5 & 26.68 & 26.70 & 25.65 & 25.66 \\ 2 & 23.05 & 24.96 & 23.36 & 23.75 \\ 3 & 22.86 & 20.76 & 19.91 & 20.56 \\ 4 & 21.87 & 19.79 & 19.45 & 19.43\end{array}$

\begin{tabular}{llccc}
\hline \multicolumn{5}{c}{ Three-group $\left(L_{1}^{2}=10 \mathrm{~cm}^{2}\right), L_{2}^{2}=\left(\mathrm{cm}^{2}\right)$} \\
1 & 20.95 & 22.35 & 24.04 & 23.85 \\
1.5 & 23.80 & 23.96 & 22.89 & 22.89 \\
2 & 20.24 & 22.16 & 20.74 & 21.06 \\
3 & 20.78 & 18.05 & 17.27 & 17.86 \\
4 & 19.21 & 17.14 & 16.90 & 16.84 \\
\hline \multicolumn{5}{c}{ Age, $M^{2}=\left(\mathrm{cm}^{2}\right)$} \\
1 & 31.90 & 33.40 & 35.83 & 35.32 \\
1.5 & 33.20 & 34.52 & 34.57 & 34.10 \\
2 & 29.91 & 32.36 & 32.30 & 32.28 \\
3 & 30.84 & 29.54 & 29.37 & 29.52 \\
4 & 30.52 & 29.26 & 29.78 & 29.36 \\
\hline
\end{tabular}

\begin{tabular}{lcrrr}
\hline \multicolumn{4}{c}{ Age-diffusion, $\tau=\left(\mathrm{cm}^{2}\right)$} \\
1 & 29.84 & 31.10 & 33.48 & 33.07 \\
1.5 & 31.34 & 32.07 & 32.06 & 31.68 \\
2 & 27.96 & 30.06 & 29.58 & 29.61 \\
3 & 28.47 & 26.58 & 26.23 & 26.53 \\
4 & 27.63 & 25.97 & 26.50 & 26.16 \\
\hline
\end{tabular}

In practice, $L_{s}{ }^{2}$ was calculated as above. Three values of $L_{1}{ }^{2}$ were tried: $5 \mathrm{~cm}^{2}, 7.5 \mathrm{~cm}^{2}$, and 10 $\mathrm{cm}^{2}$. Then $v$ was calculated, and a least-squares fit led to $L_{2}^{2}$.

$$
M^{2}=L_{s}{ }^{2}+L_{1}{ }^{2}+L_{2}^{2}
$$

Age.

$$
\varepsilon p f \eta=\exp \left(M^{2} B^{2}\right) .
$$

Thus $\ln (f \eta)$ is a linear function of $B^{2}$. A leastsquares fit of $\ln (f \eta)$ vs $B^{2}$ yields $M^{2}$ as the slope.

Age Diffusion.

$$
\varepsilon p f \eta=\left(1+L_{s}^{2} B^{2}\right) \exp \left(\tau B^{2}\right) .
$$

Therefore $\ln u$ is a linear function of $B^{2}$, and $\tau$ is the slope.

$$
M^{2}=\tau+L_{s}^{2} .
$$

The basic data for the analysis (values of $f, \eta, B^{2}$ ) are gathered in Tables 14 and 15, for the benefit of those who may wish to fit these measurements into alternative calculational schemes.

Least-squares fits to all seven critical equations were first made separately for the three situations: 1) enrichment varied, moderator unpoisoned, 2) $1.3 \%$ enriched fuel, moderator poisoned, 3) $1.15 \%$ fuel, moderator poisoned. The results of this analysis are compiled in Table 17.

This is the same sort of analysis as that presented at length in the Geneva report, ${ }^{1}$ which there indicated a superiority of an age-type critical equation over a one-group form. Now however the corrected values of thermal utilization used here have caused this apparent advantage largely to disappear. A statistical analysis shows that the three-group expressions and the age and agediffusion forms all fit the data somewhat better than do the one and two-group equations. The superiority is however not marked, and is indeed within the probable error of the experiment.

The data for each volume ratio were then combined and analyzed as a unit, with the results also shown in Table 17. From these results the values of $k_{\infty}$ given in Table 18 were calculated.

It is immediately apparent that the final values of $k_{\infty}$ do not change much with the form of analysis used to obtain them, and there is no clear tendency for any single form of critical equation to lead to results consistently greater or smaller than the others. In other words, the final values of $k_{\infty}$ 
Table 18

\begin{tabular}{ccccccccc}
\multicolumn{2}{c}{} & \multicolumn{7}{c}{$k_{\infty}$} \\
\hline $\begin{array}{c}\text { Volume } \\
\text { ratio }\end{array}$ & $\begin{array}{c}\text { One- } \\
\text { group }\end{array}$ & $\begin{array}{c}\text { Two- } \\
\text { group }\end{array}$ & $\begin{array}{c}L_{1}{ }^{2} \\
5 \mathrm{~cm}^{2}\end{array}$ & $\begin{array}{c}L_{1}{ }^{2} \\
7.5 \mathrm{~cm}^{2}\end{array}$ & $\begin{array}{c}L_{1}{ }^{2} \\
10 \mathrm{~cm}^{2}\end{array}$ & $\begin{array}{c}\text { Age } \\
\text { Agge- } \\
\text { diffusion }\end{array}$ \\
\hline & 1 & 1.118 & 1.118 & 1.119 & 1.119 & 1.119 & 1.120 & 1.120 \\
& 1.5 & 1.191 & 1.191 & 1.192 & 1.192 & 1.191 & 1.194 & 1.193 \\
& 2 & 1.217 & 1.216 & 1.216 & 1.216 & 1.216 & 1.218 & 1.217 \\
& 3 & 1.200 & 1.198 & 1.198 & 1.198 & 1.197 & 1.197 & 1.196 \\
& 4 & 1.156 & 1.157 & 1.158 & 1.158 & 1.158 & 1.159 & 1.159 \\
& 1.15 & 1.079 & 1.079 & 1.079 & 1.079 & 1.079 & 1.078 & 1.079 \\
& 1.5 & 1.148 & 1.149 & 1.148 & 1.148 & 1.148 & 1.147 & 1.148 \\
& 2 & 1.171 & 1.171 & 1.170 & 1.170 & 1.170 & 1.169 & 1.169 \\
& 3 & 1.154 & 1.154 & 1.153 & 1.152 & 1.152 & 1.149 & 1.150 \\
& 4 & 1.112 & 1.113 & 1.113 & 1.113 & 1.113 & 1.112 & 1.113 \\
& 1 & 1.037 & 1.037 & 1.037 & 1.037 & 1.036 & 1.036 & 1.036 \\
& 1.5 & 1.109 & 1.110 & 1.109 & 1.109 & 1.108 & 1.106 & 1.107 \\
& 2 & 1.128 & 1.128 & 1.127 & 1.127 & 1.127 & 1.123 & 1.125 \\
& 3 & 1.109 & 1.109 & 1.107 & 1.107 & 1.106 & 1.103 & 1.104 \\
& 4 & 1.065 & 1.066 & 1.065 & 1.065 & 1.065 & 1.063 & 1.064 \\
\hline
\end{tabular}

have turned out to be invariant of the interpretation. This result is of fundamental importance; it implies that regardless of which simplified model is chosen to approximate the behavior of this form of reactor, the non-leakage probabilities will be calculated correctly - so long as the migration area used is the one found with the same model.

The probable errors given in Table 17 were obtained from a compounding of probable errors in $B^{2}$ and $f$ in the usual way. The estimates of error in $f$ were based on the possibility that the flux average in the moderator might be incorrect by $2 \%$. This almost certainly is an overestimate of errors in $f$ from all sources.

$M^{2}$ as found from three-group theory with $L_{1}{ }^{2}=7.5 \mathrm{~cm}^{2}$ is plotted in Figure 18 . Figure 19 shows the dependence of $k_{\infty}$ on volume ratio for the three enrichments.

\section{The Value of $\boldsymbol{\eta}^{7}$}

A value of $\eta$ for natural uranium can now be derived, since everything in the four-factor formula except $\eta$ has been measured. Values of $k_{\infty}$ are chosen from Table 18, and those of $\varepsilon, p$, and $f$ from Tables 11, 12, and 6. For each lattice, then, a value of $\eta$ appropriate to the enrichment of the lattice can be found. To convert these to values of $\eta$ for natural uranium $\left(\eta_{\mathrm{nat}}\right)$, note that from Equation (23)

$$
\frac{\eta_{1 \%}}{\eta_{\text {nat }}}=\frac{1+\mathcal{N}_{\text {nat }} \sigma_{\text {abs }}^{28} / \sigma_{\text {abs }}^{25}}{1+\mathcal{N}_{1 \%} \sigma_{\text {abs }}^{28} / \sigma_{\text {abs }}^{25}}
$$

where

$\mathcal{N}_{\text {nat }}=\frac{\text { Number of } \mathrm{U}^{238} \text { nuclei/cc in natural } \mathrm{U}}{\text { Number of } \mathrm{U}^{235} \text { nuclei/cc in natural U }}=137.8$

and

$\mathcal{N}_{1 \%}=$ same ratio in $1 \%$ enriched $\mathrm{U}=95.16$.

Thus

$$
\eta_{1 \%} / \eta_{\text {nat }}=1.126 \pm 0.009
$$

and similarly

$$
\eta_{1.15 \%} / \eta_{\text {nat }}=1.159 \pm 0.009
$$

and

$$
\eta_{1.3 \%} / \eta_{\text {nat }}=1.196 \pm 0.010 .
$$

Accordingly $\eta_{\text {nat }}$ can be calculated from the average values of $\eta_{1 \%}, \eta_{1.15 \%}$, and $\eta_{1.3 \%}$ which result from the lattice measurements. For the 0.600-in. rods, these values are found to be

$$
\begin{array}{ll}
\eta_{1 \%}=1.456 & \eta_{\text {nat }}=1.293 \\
\eta_{1.15 \%}=1.512 & \eta_{\text {nat }}=1.305 \\
\eta_{1.3 \%}=1.554 & \eta_{\text {nat }}=1.299
\end{array}
$$

These results have been combined with those obtained from other rod sizes and in the BNL 


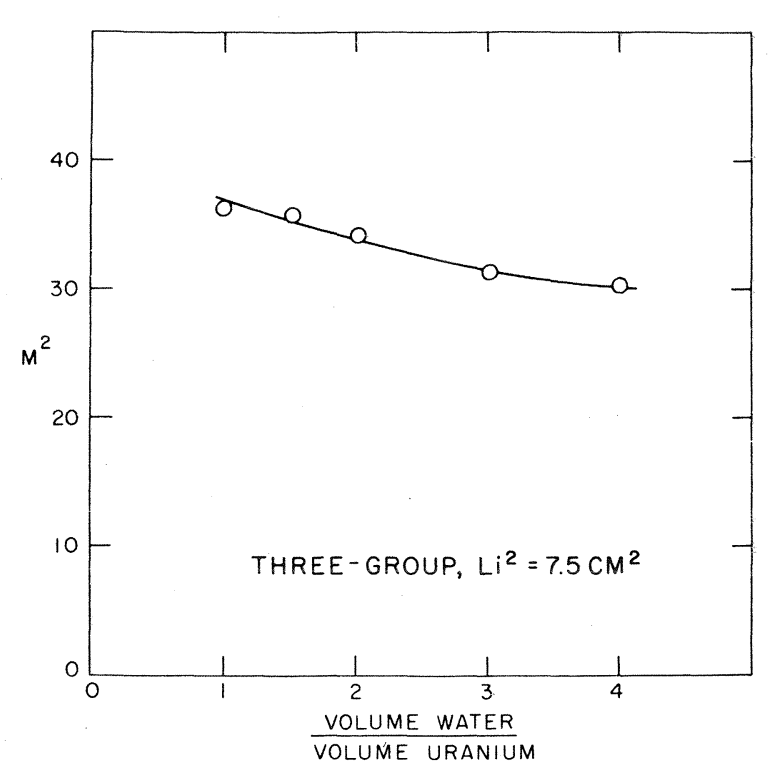

Figure 18. Migration area, 0.600 in.

natural $\mathrm{U}$, graphite moderated reactor to provide what is probably the best measured value of $\eta_{\text {nat }}$ to date, namely

$$
\eta_{\text {nat }}=1.309 \pm 0.006 \text {. }
$$

Similar measurements by Clayton and by Donohue at Hanford on uranium-graphite lattices give 1.308 and 1.313 , in excellent agreement with the above value. ${ }^{8}$

\section{The Role of Epi-Thermal \\ Fissions in Uranium-235}

An analysis of these measurements in terms of a three-group model has been made in which the effect of epi-thermal fissions in $\mathrm{U}^{235}$ is taken into account. ${ }^{9}$ As before, all $1 / v$ processes are classed as "thermal," and therefore the thermal quantities in the four-factor formula, $f$ and $\eta$, are retained. It is then possible to derive the following critical equation:

$$
\begin{aligned}
& 1=\frac{f \eta(1+\rho)}{A_{1} A_{2} A_{3}(1+x)}\left\{1+\frac{F}{\nu_{25}}\left[\left\{\nu_{28}-\left(1+\alpha_{28}\right) A_{1}\right\}+\right.\right. \\
& \left.\left.\rho\left\{\nu_{28}\left(1-A_{2}\right)-\left(1+\alpha_{28}\right)\left(A_{1}-\frac{f \eta}{A_{3}(1+x)}\right)\right\}\right]\right\}
\end{aligned}
$$

where $1 / A_{1}, 1 / A_{2}, 1 / A_{3}$ are non-leakage probabilities in the fast, resonance, and thermal regions respectively;

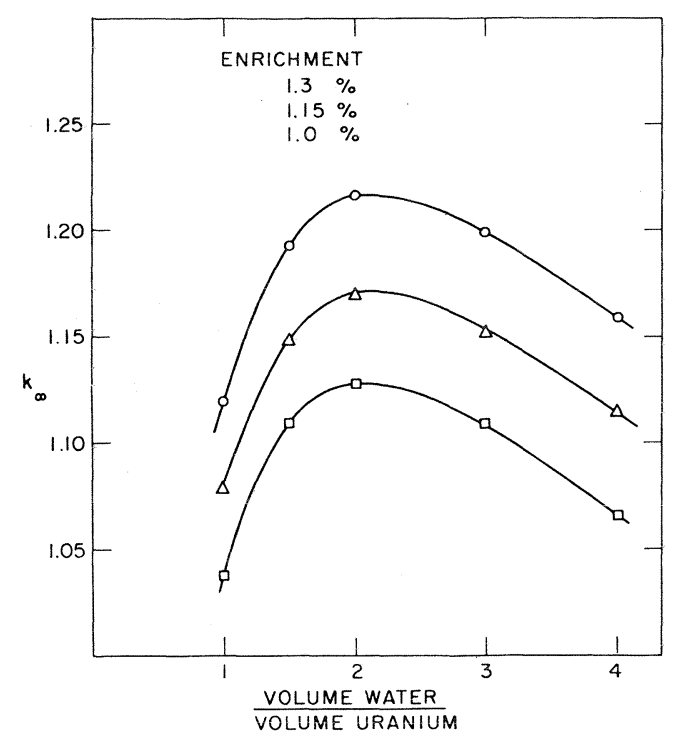

Figure 19. Dependence of $k_{\infty}$ on volume ratio.

$$
\begin{gathered}
x \equiv \frac{f}{A_{3}}\left\{\Sigma S \frac{(1-\delta / S)}{(1+\delta)}-\right. \\
\left.\frac{\eta}{\nu_{25}}\left[(1+\rho) F \alpha_{28}-\rho\left(1+\alpha_{25}\right)\right]\right\} ; \\
\rho=\frac{\text { epi-thermal fission rate in } \mathrm{U}^{235}}{\text { thermal fission rate in } \mathrm{U}^{235}} ;
\end{gathered}
$$

and $\alpha_{25}$ is the capture/fission ratio in $\mathrm{U}^{235}$ averaged over the resonance region.

The somewhat arbitrary definitions

$$
\varepsilon=1+\frac{F}{\nu_{25}}\left[\nu_{28}-\left(1+\alpha_{28}\right) A_{1}\right]
$$

and

$$
\begin{gathered}
p=\frac{(1+\rho)}{(1+x)}\left\{1+\frac{F}{\nu_{25}} \frac{\rho}{\varepsilon}\right. \\
\left.\times\left[\nu_{28}\left(1-A_{2}\right)-\left(1+\alpha_{28}\right)\left(A_{1}-\frac{f \eta}{A_{3}(1+x)}\right)\right]\right\}
\end{gathered}
$$

cause the critical equation to take on the form of the familiar four-factor formula.

In the limit $\rho=0$,

$$
\varepsilon_{0}=1+\frac{F}{\nu_{25}}\left[\nu_{28}-\left(1+\alpha_{28}\right) A_{1}\right]
$$

and

$$
p_{0}=\frac{1+\frac{F}{\varepsilon} \frac{\alpha_{28} A_{1} A_{2}}{\nu_{25}}}{1+\frac{f S \Sigma}{A_{3}} \frac{(1-\delta / S)}{(1+\delta)}}
$$


These are the expressions mentioned in section $\mathrm{V}$-A that differ slightly from Equations (16) and (17) used in the four-factor interpretation.

The three-group form of the critical equation can be used to calculate $\eta$, since everything else is known except the non-leakage probabilities $A_{1}, A_{2}, A_{3}$, for which reasonable estimates can be made. The values of $\eta$ so calculated, in terms of $\rho$ and the primary measured quantities $F$ and $S$ differ only slightly of course from those calculated in section $\mathrm{V}-\mathrm{C}$, since $\rho$ is so small. (It should be remembered that $\rho$ is the epi-thermal, i.e., non$1 / v$, fission fraction in $\mathrm{U}^{235}$ which is a small fraction of the epi-Gd fraction, which is itself quite small.)

\section{E. Conversion Ratios}

The initial (clean, cold) conversion ratios in these lattices can be easily inferred from the data given in earlier sections. Appendix IV derives expressions for the conversion ratio in terms of measured quantities. These expressions are

$$
C=\frac{(1+S)}{(1+\delta)} \frac{\nu_{25} \Sigma}{\eta(1+\alpha)\left[1+\beta \rho \rho_{m} /\left(1-\rho \rho_{m}\right)\right]}
$$

and, alternatively,

$$
C=\frac{(1+S) \nu_{25} \Sigma}{\eta(1+\alpha)\left[1+\rho \rho_{m}(\beta-1)\right] /\left(1-\rho \rho_{m}\right)}
$$

where the symbols are defined in Appendix IV. The value of $C$ computed by Equation (34) is denoted by $C_{\delta}$, and that computed by Equation (35) by $C_{\rho_{m}}$. In Appendix IV are tabulated the values of $C_{\delta}$ and $C_{\rho_{m}}$. $C_{\delta}$ should of course equal $C_{\rho_{m}}$; the disagreements between their tabulated values imply that the values of $\delta$ and/or $\rho_{m}$ reported here are incorrect. The values of $\rho_{m}$ are in general considerably lower than those measured in similar (critical) lattices at Westinghouse (Bettis), ${ }^{6}$ whereas the Westinghouse values would considerably reduce the disagreements between $C_{\delta}$ and $C_{\rho_{m}}$; it is on this basis that the statement is made in section IV-H that Brookhaven values of $\rho_{m}$ are probably faulty.

\section{F. The Effect on $\varepsilon$ of Finite Lattice Size}

It has been pointed out long ago by R. Hellens ${ }^{10}$ and more recently by S. Stein ${ }^{11}$ that leakage in the energy region of fast fission can materially influence the value of $\varepsilon$. This effect can be gauged by the application of a three-group theory, in which the boundary between the fast and intermediate energy groups is the $\mathrm{U}^{238}$ fast fission threshold, and the intermediate and thermal groups are divided in the usual way. This model has been developed in Appendix VI, where it is shown that the critical equation modified to allow for fast leakage assumes the form

where

$$
A_{s} A_{1} A_{2}=\varepsilon p f \eta
$$

$$
\varepsilon=1+F \frac{\nu_{28}-A_{1}(1+\alpha)}{\nu_{25}}
$$

and the $A_{i}$ are the reciprocals of the non-leakage probabilities in the separate groups:

$$
A_{i}=1+L_{i}{ }^{2} B^{2}=\mathcal{L}_{i}{ }^{-1} .
$$

The value of $k_{\infty}$ is

where

$$
k_{\infty}=\varepsilon_{\infty} p f \eta
$$

$$
\begin{gathered}
\varepsilon_{\infty}=1+F_{\infty}\left[\frac{\nu_{28}-(1+\alpha)}{\nu_{25}}\right], \\
\frac{F}{F_{\infty}}=\mathcal{L}_{1}-F \frac{\nu_{28}}{\nu_{25}}\left(1-\mathcal{L}_{1}\right) \cong \mathcal{L}_{1} .
\end{gathered}
$$

Thus $\varepsilon$ and $\varepsilon_{\infty}$ are not the same. Actually, it follows from (36), (37), and (38) that

$$
(\varepsilon-1) \cong\left(\varepsilon_{\infty}-1\right) \mathcal{L}_{1}\left[1-\frac{\left(A_{1}-1\right)(1+\alpha)}{\nu_{28}-(1+\alpha)}\right] .
$$

Therefore $(\varepsilon-1)$ in a finite assembly (non-vanishing $\left.B^{2}\right)$ is reduced from that in an infinite array by the factor

$$
K \cong \mathcal{L}_{1}\left[1-\frac{\left(A_{1}-1\right)(1+\alpha)}{\nu_{28}-(1+\alpha)}\right] .
$$

In order to estimate the size of the reduction, it is necessary to have information on the magnitude of $\mathcal{L}_{1}$. The following considerations should be reasonably correct.

According to the Geneva paper of E. Wilkins et al., ${ }^{12}$ the value of $\bar{r}^{2} / 6$ around a point source of fission neutrons in water is about $18 \mathrm{~cm}^{2}$ at 330 kev. A reasonable value at $1 \mathrm{Mev}$ (nominal $\mathrm{U}^{238}$ fission threshold) is probably about $15 \mathrm{~cm}^{2}$. The calculation in this Geneva paper leads to a value of $\bar{r}^{2} / 6=25.3 \mathrm{~cm}^{2}$ at $1.44 \mathrm{ev}$, and the slowing down from $1.44 \mathrm{ev}$ to thermal should add about $1 \mathrm{~cm}^{2}$. The division of the age in pure water between the fast and intermediate groups is therefore approximately 
Table 19

Decomposition of Leakage

\begin{tabular}{cccccrcc}
\hline \multirow{2}{*}{ Enrichment } & $\begin{array}{c}\text { Volume } \\
\text { ratio }\end{array}$ & $M^{2}$ & $L_{s}{ }^{2}$ & $L_{2}{ }^{2}$ & $L_{1}{ }^{2}$ & $A_{1}$ & $\mathcal{L}_{1}$ \\
\hline \multirow{2}{*}{1.3} & 1 & 36.1 & 2.2 & 24.5 & 9.4 & 1.030 & 0.971 \\
& 1.5 & 35.3 & 2.4 & 20.0 & 12.9 & 1.067 & 0.937 \\
& 2 & 33.6 & 2.6 & 17.8 & 13.2 & 1.081 & 0.925 \\
& 3 & 30.8 & 2.9 & 15.5 & 12.4 & 1.076 & 0.929 \\
& 4 & 30.1 & 3.2 & 14.4 & 12.5 & 1.063 & 0.941 \\
1.15 & 1 & 36.3 & 2.4 & 24.5 & 9.4 & 1.020 & 0.980 \\
& 1.5 & 35.5 & 2.6 & 20.0 & 12.9 & 1.052 & 0.951 \\
& 2 & 33.8 & 2.8 & 17.8 & 13.2 & 1.064 & 0.940 \\
& 3 & 31.0 & 3.1 & 15.5 & 12.4 & 1.058 & 0.945 \\
1 & 4 & 30.3 & 3.4 & 14.4 & 12.5 & 1.045 & 0.957 \\
& 1 & 36.4 & 2.6 & 24.5 & 9.3 & 1.009 & 0.991 \\
& 1.5 & 35.7 & 2.8 & 20.0 & 12.9 & 1.038 & 0.963 \\
& 2 & 34.0 & 3.0 & 17.8 & 13.2 & 1.048 & 0.954 \\
& 3 & 31.2 & 3.3 & 15.5 & 12.4 & 1.041 & 0.961 \\
& 4 & 30.5 & 3.7 & 14.4 & 12.4 & 1.026 & 0.975 \\
\hline
\end{tabular}

$\begin{array}{ll}\text { Fast } & 15 \mathrm{~cm}^{2} \\ \text { Intermediate } & 11 \mathrm{~cm}^{2}\end{array}$

In water-uranium lattices, where an appreciable fraction of the moderator is displaced by metal, this division must be changed. In the intermediate region, uranium inelastic scattering is small, and only the hydrogen density should account for slowing down. The effective logarithmic decrement in this region is therefore reduced by approximately a factor

$$
\frac{V_{w}}{V_{w}+V_{u}+V_{a l}}
$$

and the intermediate age $L_{2}{ }^{2}$ by the reciprocal of this, or about

$$
\frac{V_{w}+V_{u}+V_{a l}}{V_{w}}=1+\frac{V_{u}}{V_{w}}+\frac{V_{a l}}{V_{w}} .
$$

The value of this varies from 2.23 at the $1: 1 \mathrm{vol}-$ ume ratio to 1.31 at the $4: 1$ volume ratio. Thus, by the above assumptions, the intermediate age should vary from about $24.5 \mathrm{~cm}^{2}$ to $14.4 \mathrm{~cm}^{2}$ within this range.

The $M^{2}$ and $L_{s}^{2}$ previously found can now be used to obtain approximate values of $L_{1}{ }^{2}$; the results of these calculations for the 15 clean lattices are listed in Table 19. It must be stressed that these estimates are only approximate, and are being used for order-of-magnitude purposes.
Table 11, presented earlier, lists values of $\varepsilon$ as calculated from Equation (16). It should be noted, however, that Equation (16) and Equation (36) are not identical, and therefore the values in Table 11 are not those of $\varepsilon$ in a finite system. They can however be corrected to either the finite or the infinite values through use of the preceding analysis; the results of doing so are seen in Tables 20 and 21 .

However $\varepsilon$ contains still another uncertainty, i.e., the unknown value of $\nu_{28}$. Since the degree of error from this source is still only a matter for speculation, it has not been thought worth while to pursue the effects of fast leakage on the final values beyond what has been given above.

\section{G. The Effect of Fast Leakage on the $M^{2}$ Determination}

In the analysis of the data to find the migration area, it was assumed that the only intensive quantities which varied with poison or enrichment were $f$ and $\eta$. The preceding section has however been concerned with another effect, which, though small, must also be taken into account. This is the variation of the finite system value of $\varepsilon$.

The size of the error introduced by neglecting this can be derived in a straightforward way. The three-group critical equation derived in Appendix $\mathrm{VI}$ is 
Table 20

\begin{tabular}{lccc}
\multicolumn{4}{c}{$(\varepsilon-1)_{\infty}$} \\
\hline $1.3 \%$ & $1.15 \%$ & $1.0 \%$ & Average \\
\hline 0.102 & 0.100 & 0.106 & 0.103 \\
0.080 & 0.080 & 0.084 & 0.081 \\
0.068 & 0.067 & 0.069 & 0.068 \\
0.053 & 0.048 & 0.053 & 0.051 \\
0.045 & 0.043 & 0.041 & 0.043 \\
\hline
\end{tabular}

where

$$
A_{1} A_{2} A_{s}=\varepsilon p f \eta
$$

$$
\begin{aligned}
\varepsilon & =1+F \frac{\nu_{28}-A_{1}(1+\alpha)}{\nu_{25}} \\
& \cong 1+(\varepsilon-1)_{\infty} \mathcal{L}_{1} C .
\end{aligned}
$$

The three-group migration area analysis has been based on assuming $L_{1}{ }^{2}$ and $L_{s}{ }^{2}$ known, and supposing that a linear relationship holds between $B^{2}$ and

$$
v=f \eta / A_{1} A_{s}
$$

this relationship being

$$
1+L_{2}^{2} B^{2}=\varepsilon p v .
$$

The variation also of $\varepsilon$ however means that $B^{2}$ is linearly related to $w$, where

such that

$$
w=f \eta \varepsilon / A_{1} A_{s}
$$

$$
1+L_{2}^{2} B^{2}=p w .
$$

From Equation (40),

$$
L_{2}^{2}=\left(\frac{1}{v}\right)_{B^{2}=0} \frac{d v}{d B^{2}} .
$$

However, from Equation (41),

$$
\left(L_{2}^{2}\right)^{*}=\left(\frac{1}{w}\right)_{B^{2}=0} \frac{d w}{d B^{2}} .
$$

The asterisk has been added to allow for the difference in values of the diffusion area as found from the two equations.

By definition,

$$
\begin{aligned}
\frac{d w}{d B^{2}} & =\varepsilon \frac{d U}{d B^{2}}+U \frac{d \varepsilon}{d B^{2}} \\
& =\varepsilon \frac{d U}{d B^{2}}+U \frac{d}{d B^{2}}(\varepsilon-1) .
\end{aligned}
$$

Table 21

\begin{tabular}{ccc} 
& $(\varepsilon-1)_{\text {finite }}$ & \\
\hline $1.3 \%$ & $1.15 \%$ & $1.0 \%$ \\
\hline 0.097 & 0.095 & 0.104 \\
0.071 & 0.073 & 0.079 \\
0.059 & 0.060 & 0.064 \\
0.046 & 0.043 & 0.049 \\
0.040 & 0.040 & 0.039 \\
\hline
\end{tabular}

From Equation (39) and the definition of $C$ in Appendix VI,

$$
\frac{d}{d B^{2}}(\varepsilon-1) \cong-\frac{\nu_{28}}{\nu_{28}-(1+\alpha)} L_{1}^{2} \mathcal{L}_{1}(\varepsilon-1)_{\infty} .
$$

Insertion of sample values in (42) shows that

$$
\frac{d}{d B^{2}}(\varepsilon-1)=-0.03 \frac{d v}{d B^{2}}
$$

It follows then from Equations (42) and (43) that $\left(L_{2}{ }^{2}\right)^{*}$ is smaller than $L_{2}{ }^{2}$ by about $3 \%$, and therefore that taking into account the effect of fast leakage reduces the deduced migration areas by amounts smaller than $2 \%$ of their previous values. This change is less than the probable error of the measurement, and, since the estimate is only approximate, it has been ignored.

It must be noted, however, that the values of $k_{\infty}$ reported in Table 18 would not agree with

$$
k_{\infty}=\varepsilon_{\infty} p f \eta
$$

and that according to Appendix VI,

$$
k_{\infty} \neq A_{1} A_{2} A_{s} .
$$

Rather, Equations (2) and (12) of Appendix VI lead to

$$
k_{\infty}=\frac{\varepsilon_{\infty}}{\varepsilon} A_{1} A_{2} A_{s}
$$

\section{SUMMARY AND CONCLUSIONS}

The data published in this report and their interpretation are in as final a form as knowledge of the nuclear parameters permits.

The values of buckling are apparently correct to within their cited probable errors. The previously published thermal utilizations have been corrected by removal of a systematic error associated with technique, and the new values are be- 
lieved to be trustworthy. Values of the fast-to-thermal fission ratio have been found for all 15 clean lattices. A number of measurements of resonance absorption in $\mathrm{U}^{238}$ have been carried out; their results are in reasonable agreement with similar ones found elsewhere. Epi-cadmium fission fractions have been measured also, but although these are reported here, they are believed to be incorrect.

The analysis for the migration area has been extended in two ways: more data have been added, and also the consequences of varying the nature of the analysis have been investigated. The results have not led to clarification of the precise values of $M^{2}$, but they have shown that the $k_{\infty}$ are probably correct (subject to a proper interpretation of the effects of fast leakage). The use of corrected values of $f$ in this study leads to three-group values of $M^{2}$ which are in slightly better agreement with those measured in Russia. ${ }^{13}$ The migration areas reported here seem to agree reasonably with those found at WAPD, ${ }^{5}$ when allowance is made for uncertainties imposed on the latter by inadequate knowledge of the inhour equation.

Although the lack of an experimental value of $\nu_{28}$ limits the extent to which fast fission measurements can be interpreted through $\varepsilon$, the quantity really needed for the neutron economics of longterm behavior is the measured $F$ itself. Therefore, although it remains important for $\nu_{28}$ to be found, the immediate needs in this respect for reactor design seem to be met by the experiments.

On the other hand, uncertainty in $\varepsilon$ is reflected in the values of $\eta$ deduced from the complete study. The value of $\eta$ must be known for reactor core life predictions, and so the determination of $\nu_{28}$ is most important for this reason.

However, the close agreement between $\eta$ for natural uranium as deduced from water lattice experiments and on the other hand from evaluation of the parameters of the Brookhaven pile implies that the assumption $\nu_{28}=2.5$ cannot be grossly in error.

\section{ACKNOWLEDGMENTS}

It would be impossible to acknowledge adequately the many people who contributed to this work. The BNL experimental reactor physics group included, besides the authors, K.W. Downes,
G.A. Price, V. Walsh, L. Heintze, A. Tierney, Helen Connell, and Joyce Meyer, and several visiting scientists and summer students, as well as several technicians. Conversations with Irving Kaplan and with Jack Chernick and others in the theoretical reactor physics group have always been particularly valuable. There have also been stimulating and friendly talks with members of the Westinghouse Bettis group who have had closely related interests. We also wish to take this opportunity of thanking the BNL Reactor Operations Division for their constant and willing cooperation. Finally, a word of appreciation is in order to two groups whose excellent and prompt assistance was vital to the measurements: the analytical chemistry group under R.W. Stoenner, and the hot machine shop under A. Roy.

\section{REFERENCES}

1. H. Kouts, Proc. Intern. Conf. on Peaceful Uses of Atomic Energy, UN, New York, 1956, Paper $\mathrm{P} / 600$.

2. R.L. Zimmerman, Private communication.

3. H. Kouts et al., A Measurement of Neutron Temperature in a Uranium Rod - Water Moderated Lattice, BNL-1986, Office of Technical Services, Department of Commerce, Washington 25, D.C.

4. K. Downes and H. Kouts, Reactivity Coefficient Measurement of Buckling, BNL-1785, Office of Technical Services, Department of Commerce, Washington 25, D.C.

5. S. Krasik and A. Radkowsky, Proc. Intern. Conf. on Peaceful Uses of Atomic Energy, UN, New York, 1956, Paper P/601.

6. D. Klein, Private communication.

7. R. Sher, Internal memorandum, BNL-402-I15, 1956.

8. Private communications.

9. R. Sher and H. Kouts, Internal memorandum, BNL-410-I-17, 1956.

10. Private communications.

11. Private communications.

12. E. Wilkins et al., Proc. Intern. Conf. on Peaceful Uses of Atomic Energy, UN, New York, 1956, Paper $\mathrm{P} / 597$.

13. L.M. Barkov, A.P. Venediktov, and K.N. Mukhin, J. Nuclear Energy 4, 103 (1957). 


\section{APPENDIX I \\ Separation of Uranium From Its Fission Products by the Sodium Uranyl Acetate Method}

This method is based upon the procedure given in CN-391, 1942. We are indebted to H.L. Finston and Mary Kinsley of the BNL Hot Laboratory for their advice and help in this portion of the work.

1. Dissolve the uranium foil in concentrated, hot nitric acid. This is to be heated in a $50-\mathrm{ml}$ centrifuge tube. When the uranium sample is completely dissolved (10 min to $1 \mathrm{hr}$ ), the remaining acid should be evaporated until an orange powder remains at the bottom of the tube.

2. Add at most $50 \mathrm{ml}$ distilled water for each gram uranium dissolved. It may be necessary to heat this solution gently to dissolve the uranyl nitrate completely.

3. From the solution obtained in step 2, pipette by syringe $3 \mathrm{ml}$ to another tube and add $1 \mathrm{ml}$ glacial acetic acid. Heat this solution gently to evaporate down to $1 / 2 \mathrm{ml}$.

4. To this solution add $1 \mathrm{ml}$ (20 drops) $1 M$ acetic acid and $1 \mathrm{ml} 1 M$ sodium acetate and shake. A precipitate should begin to settle at this point. If not, warm gently to concentrate the solution.

5. Add 21/2 ml (50 drops) $5 \mathrm{M} \mathrm{NaNO}_{3}$.

6. Cool in ice water and centrifuge for $1 \mathrm{~min}$.

7. Pour off the supernatant liquid and dissolve the yellow precipitate in 1 drop concentrated nitric acid.

8. Repeat steps 4 to 7 two more times, making a total of three.

9. After the third centrifuging, do not add nitric acid after pouring off the supernatant, but pour the precipitate onto a filter paper which has been dried with ether. The precipitate can be washed from the tube by using cold alcohol.

10. Wash the yellow filtrate with a little cold alcohol and a few drops cold ether.

11. Dry the powder on the filter paper and then scrape into a 10-ml beaker. Any desired amount of water can then be added.

12. Pipette or pour solution onto clean planchets. 13. Slowly dry the planchets under a heat lamp. 


\section{APPENDIX II}

\section{The Effect of Neutron Temperature on $f$}

\section{A. The Nature of an $f$ Measurement}

The measurement of thermal utilization is done by exposing foils of dysprosium oxide in the moderator and in the uranium, to provide a spacial distribution of what is loosely called the "flux." What is actually obtained by this process is the curve of virtual capture density in dysprosium throughout a lattice cell. The difference can be seen by the following analysis. Let

$$
\begin{aligned}
\phi_{m}(E, x) & =\text { flux in water, } \\
\phi_{u}(E, x)= & \text { flux in uranium, } \\
\sigma_{D}(E)= & \text { dysprosium cross section, and } \\
\mathcal{N}_{D}= & \text { number of dysprosium atoms } \\
& \text { in a standard foil. }
\end{aligned}
$$

Then the saturated activity of a dysprosium foil at the position $x$ in the moderator is

$$
A_{m}(x)=\int d E \mathcal{N}_{D} \sigma_{D}(E) \phi_{m}(E, x)
$$

and that in the fuel is

$$
A_{u}(x)=\int d E \mathcal{N}_{D} \sigma_{D}(E) \phi_{u}(E, x)
$$

These then are the basic measured quantities. If dysprosium had a strictly $1 / v$ cross section, the curves of $A_{m}(x)$ and $A_{u}(x)$ would give the distribution of total neutron density.

The analysis used to deduce the thermal utilization proceeds thus (the aluminum cladding is neglected). Define the integrals

$$
\begin{aligned}
& I_{m}=\int_{\text {mod }} d x A_{m}(x)=\int_{\text {mod }} d x \int d E \mathcal{N}_{D} \sigma_{D}(E) \phi_{m}(E, x), \\
& (\mathrm{A} 2-2 \mathrm{a}) \\
& I_{u}=\int_{\text {fuel }} d x A_{u}(x)=\int_{\text {fuel }} d x \int d E \mathcal{N}_{D} \sigma_{D}(E) \phi_{u}(E, x) .
\end{aligned}
$$

Now let
$\sigma_{m}(E)=$ absorption cross section of a moderator atom,

$\sigma_{u}(E)=$ absorption cross section of a uranium atom,

$\mathcal{N}_{m}=$ atoms $/ \mathrm{cm}^{3}$ of moderator, and

$\mathcal{N}_{u}=$ atoms $/ \mathrm{cm}^{3}$ of uranium.

Then the capture density in the moderator is at $x$

$$
C_{m}(x)=\int d E \sigma_{m}(E) \mathcal{N}_{m} \phi_{m}(E, x) .
$$

In the fuel, the capture density at $x$ is

$$
C_{u}(x)=\int d E \sigma_{u}(E) \mathcal{N}_{u} \phi_{u}(E, x) .
$$

The total capture rates in the moderator and fuel are, respectively,

$J_{m}=\int_{\bmod } d x C_{m}(x)=\int_{\bmod } d x \int d E \sigma_{m}(E) \mathcal{N}_{m} \phi_{m}(E, x)$

$J_{u}=\int_{\text {fuel }} d x C_{u}(x)=\int_{\text {fuel }} d x \int d E \sigma_{u}(E) \mathcal{N}_{u} \phi_{u}(E, x)$

Hence the thermal utilization is given by

$$
f=\frac{J_{u}}{J_{m}+J_{u}} .
$$

If the cross sections of moderator, fuel, and dysprosium all had the same energy dependence it would follow that

$$
\begin{aligned}
& \frac{\sigma_{m}(E)}{\sigma_{D}(E)}=\frac{\sigma_{m}{ }^{0}}{\sigma_{D}{ }^{0}}, \\
& \frac{\sigma_{u}(E)}{\sigma_{D}(E)}=\frac{\sigma_{u}{ }^{0}}{\sigma_{D}{ }^{0}}
\end{aligned}
$$

where the superscript 0 implies evaluation at 2200 $\mathrm{m} / \mathrm{sec}$

The use of $(6 a),(6 b),(2 a)$, and (2b) would permit (4a) and (4b) to be written as 


$$
\begin{aligned}
J_{m} & =\frac{\mathcal{N}_{m} \sigma_{m}^{0}}{\mathcal{N}_{D} \sigma_{D}} \int_{\text {mod }} d x \int d E \sigma_{D}(E) \mathcal{N}_{D} \phi_{m}(E, x) \\
& =\frac{\mathcal{N}_{m} \sigma_{m}{ }^{0}}{\mathcal{N}_{D} \sigma_{D}{ }^{0}} I_{m} \\
J_{u} & =\frac{\mathcal{N}_{u} \sigma_{u}^{0}}{\mathcal{N}_{D} \sigma_{D}^{0}} \int_{\text {fuel }} d x \int d E \sigma_{D}(E) \mathcal{N}_{D} \phi_{u}(E, x) \\
& =\frac{\mathcal{N}_{u} \sigma_{u}{ }^{0}}{\mathcal{N}_{D} \sigma_{D}{ }^{0}} I_{u} .
\end{aligned}
$$

Therefore Equation (5) becomes

$$
f=\frac{\mathcal{N}_{u} \sigma_{u}{ }^{0} I_{u}}{\mathcal{N}_{u} \sigma_{u}{ }^{0} I_{u}+\mathcal{N}_{m} \sigma_{m}{ }^{0} I_{m}}
$$

The analysis up to and including Equation (5) is precise. That in Equations (6), (7), and (8) depends on the assumption that the three substances concerned have identically energy dependent cross sections.

\section{B. Effects of Cross Section and Neutron Temperature Differences}

Actually there are differences in the way the cross sections of dysprosium, uranium, and moderators behave with energy, and the effects of this point on the interpretation of the measurement will now be considered.

It will be noted that the deductions in the preceding section have been independent of the effective neutron temperature, and have not even depended on the assumption that the neutron temperature is the same in the fuel and the moderator. However, it will now be found that the effects of varying neutron temperature and cross section behavior are interlinked.

To simplify the analysis, it is assumed that the flux densities in fuel and moderator are separable:

$$
\begin{gathered}
\phi_{m}(E, x)=U_{m}(E) w_{m}(x), \\
\phi_{u}(E, x)=U_{u}(E) w_{u}(x) .
\end{gathered}
$$

Therefore (2a) becomes

$$
I_{m}=\mathcal{N}_{D} \int_{\bmod } d x w(x) \int d E \sigma_{D}(E) U_{m}(E) .
$$

Suppose $U_{m}(E)$ can be characterized by a Maxwell distribution with a most probable velocity $v^{\prime}$ and a dysprosium cross section $\sigma_{D}{ }^{\prime}$ at this velocity. Then (10) becomes

$$
\begin{gathered}
I_{m}=\mathcal{N}_{D} \int_{\bmod } d x w(x) \frac{\int d E \sigma_{D}(E) U_{m}(E)}{\int d E\left(\sigma_{D}{ }^{\prime} v^{\prime} / v\right) U_{m}(E)} \\
\times \int d E \frac{\sigma_{D}{ }^{\prime} v^{\prime}}{v} U_{m}(E) .
\end{gathered}
$$

But the ratio of the two integrals is just the $f$-factor for dysprosium in the energy distribution $U_{m}(E)$. This is written as $f_{D}\left(v^{\prime}\right)$ to show that it is related to the distribution with the most probable velocity $v^{\prime}$. Then

$$
I_{m}=\mathcal{N}_{D} f_{D}\left(v^{\prime}\right) \int d x w(x) \int d E\left(\sigma_{D}^{\prime} v^{\prime} / v\right) U_{m}(E) .
$$

Similarly, (4a) can be rewritten

$$
J_{m}=\mathcal{N}_{m} f_{m}\left(v^{\prime}\right) \int d x w(x) \int d E\left(\sigma_{m}^{\prime} v^{\prime} / v\right) U_{m}(E)
$$

where $f_{m}\left(v^{\prime}\right)$ and $\sigma_{m}{ }^{\prime}$ are respectively the $f$-factor and cross section of the moderator at the velocity $v_{m}{ }^{\prime}$. Thus

$$
J_{m}=\frac{\mathcal{N}_{m}}{\mathcal{N}_{D}} \frac{f_{m}\left(v^{\prime}\right)}{f_{D}\left(v^{\prime}\right)} \frac{\sigma_{m}{ }^{\prime}}{\sigma_{D}{ }^{\prime}} I_{m} .
$$

If the neutron distribution in the fuel is separable, and is characterized by a Maxwell temperature with the most probable velocity $v^{\prime \prime}$, then (4b) becomes

$$
J_{u}=\frac{\mathcal{N}_{u}}{\mathcal{N}_{D}} \frac{f_{u}\left(v^{\prime \prime}\right)}{f_{D}\left(v^{\prime \prime}\right)} \frac{\sigma_{u}{ }^{\prime \prime}}{\sigma_{D}{ }^{\prime \prime}} I_{u}
$$

On insertion of (14) and (15), Equation (5) becomes

$f=\frac{\mathcal{N}_{u} f_{u}\left(v^{\prime \prime}\right) I_{u}\left(\sigma_{u}{ }^{\prime \prime} /{\sigma_{D}}^{\prime \prime}\right)}{\left.\mathcal{N}_{u} f_{u}\left(v^{\prime \prime}\right) I_{u}\left(\sigma_{u}{ }^{\prime \prime} / \sigma_{D}{ }^{\prime \prime}\right)+\mathcal{N}_{m} f_{m}\left(v^{\prime}\right) I_{m}\left(\sigma_{m}{ }^{\prime} / \sigma_{D}{ }^{\prime}\right)\left[f_{D}\left(v^{\prime \prime}\right) / f_{D} / v^{\prime}\right)\right]}$.

The analysis of the data has been based on the expression

$$
f=\frac{\mathcal{N}_{u} f_{u}\left(v^{\prime}\right) \sigma_{u}{ }^{\prime} I_{u}}{\mathcal{N}_{u} f_{u}\left(v^{\prime}\right) \sigma_{u}{ }^{\prime} I_{u}+\mathcal{N}_{m} f_{m}\left(v^{\prime}\right) \sigma_{m}{ }^{\prime} I_{m}} .
$$

Equations (16) and (17) are identical if

$$
\begin{gathered}
\sigma_{u}{ }^{\prime \prime} / \sigma_{D}{ }^{\prime \prime}=\sigma_{u}{ }^{\prime} / \sigma_{D}{ }^{\prime}, \\
f_{D}\left(v^{\prime \prime}\right)=f_{D}\left(v^{\prime}\right), \\
f_{u}\left(v^{\prime \prime}\right)=f_{u}\left(v^{\prime}\right) .
\end{gathered}
$$




\section{Numerical Results}

The errors introduced by using Equation (A2-17) instead of Equation (A2-16) are now computed for a 3:1 volume ratio lattice of 0.600 in.-diameter, $1.3 \%$ enriched uranium rods in light water. The neutron temperatures are not precisely known, but the available evidence indicates that $T^{\prime}=300^{\circ}$ and $T^{\prime \prime}=340^{\circ}$ are not unreasonable.

Inspection of the cross section curves shows that absorption by dysprosium is decidedly not $1 / v$ in nature in the thermal region. The natural element is a mixture of seven isotopes, the one whose activation is used in the experiments having an atomic weight 164 . The low energy resonances have however been assigned ${ }^{2}$ to isotopes with weights 161 , 162, and 163. Therefore $\mathrm{Dy}^{164}$ should have a $1 / v$ cross section at low energies. This assumption has been made in the following.

Then

$$
\begin{array}{ll}
k T^{\prime} & =0.02584 \mathrm{ev} \\
v^{\prime} & =2.223 \times 10^{5} \mathrm{~cm} / \mathrm{sec} \\
k T^{\prime \prime} & =0.02929 \mathrm{ev} \\
v^{\prime \prime} & =2.367 \times 10^{5} \mathrm{~cm} / \mathrm{sec}
\end{array}
$$

$$
\begin{aligned}
& f_{u}\left(v^{\prime}\right)=0.985 \\
& f_{u}\left(v^{\prime \prime}\right)=0.984 \\
& \sigma_{u}{ }^{\prime}=11.75 \text { barns } \\
& \sigma_{u}{ }^{\prime \prime}=10.98 \text { barns } \\
& \sigma_{D}{ }^{\prime}=914 \text { barns (precise value unimportant) } \\
& {\sigma_{D}{ }^{\prime \prime}}^{\prime}=858 \text { barns }
\end{aligned}
$$

Then

$$
\begin{aligned}
& \sigma_{u}{ }^{\prime \prime} / \sigma_{D}{ }^{\prime \prime}=1.280 \times 10^{-2} \\
& \sigma_{u}{ }^{\prime} / \sigma_{D}{ }^{\prime}=1.286 \times 10^{-2}
\end{aligned}
$$

The $f$-factor for dysprosium has been assumed to be unity.

The thermal utilization calculated from Equation (A2-17) and the observed flux curves is then

$$
0.8447 \text {. }
$$

That from the use of Equation (A2-16) is

$$
0.8454 \text {. }
$$

The effect is very small, and is altogether negligible. 


\section{APPENDIX III}

\section{Subtraction of $1 / v$ Contributions From Epi-cadmium Activations}

Assume a flux distribution given by

$$
\begin{array}{ll}
\phi(E)=A M(E), & E<E_{0} ; \\
\phi(E)=B / E, & E>E_{0}
\end{array}
$$

where $M(E)=$ normalized Maxwell distribution.

The sub-Cd activation per $\mathrm{cm}^{3}$ is given by

$$
\int_{0}^{E_{0}} d E A M(E) \frac{\sigma_{0}{ }^{x}}{E^{1 / 2}}+\int_{E_{0}}^{E_{c}} d E \frac{B}{E} \frac{\sigma_{0}^{x}}{E^{1 / 2}}
$$

where $E_{c}=0.4 \mathrm{ev}$ and $\sigma_{0}{ }^{x}=$ microscopic cross section of material $x$ at $E=1 \mathrm{ev}$.

The epi-Cd activation per $\mathrm{cm}^{3}$ is

$$
\int_{E_{c}}^{\infty} d E \frac{B}{E} \frac{\sigma_{0}^{x}}{E^{1 / 2}}+\int_{\mathrm{res}} d E \frac{B}{E} \sigma_{\mathrm{res}}^{x} .
$$

The first term is the $1 / v$ activation; the second integral is the non $-1 / v$ resonance activation.*

Let

$$
\gamma_{x}=\text { ratio of } \frac{\text { epi-Cd activation }}{\text { sub-Cd activation }}
$$

in substance $x$, then

$$
\gamma_{x}=\frac{B}{A} \frac{\int_{E_{c}}{ }^{\infty} \sigma_{0}{ }^{x} d E / E^{3 / 2}+\int_{\mathrm{res}} \sigma_{\mathrm{res}}^{x} d E / E}{\int_{0}^{E_{0}} M(E) \sigma_{0}{ }^{x} d E / E^{1 / 2}+\int_{E_{0}}^{E_{c}} \sigma_{0}{ }^{x} d E / E^{3 / 2}} .
$$

Assume that the second term in the denominator can be neglected, then consider two possibilities:

1) resonance integral large compared to epi-Cd $1 / v$ integral;

2) resonance integral small compared to epi-Cd $1 / v$ integral.

*It is assumed that the epi-Cd activation can be written as the sum of $1 / v$ processes and non $-1 / v$ processes. In $\mathrm{U}^{235}$, the fission cross section just above Cd cutoff decreases faster than $1 / v$, so that the epi-Cd fission rate is not given by such a sum, in the sense that the resonance integral would be equal to that calculated from individual level parameters. However it is possible to take the difference between the total epi-Cd activation and that which would result from $1 / v$ processes (based on $\sigma_{\mathrm{th}}=580$ barns) and call this quantity the "non- $1 / v$ " contribution. For $\mathrm{U}^{235}$ this non- $1 / v$ contribution will not equal the usual resonance integral based on level parameters. It is further assumed here that the sub-Cd cross section is $1 / v$; for the purposes of this discussion this assumption is sufficiently valid.
Case 1 ) is typified by $\mathrm{Au}^{197}$, case 2) by $\mathrm{U}^{235}$. For case 1) (assume $\int_{0} E_{0} \cong \int_{0} E_{c}$ )

$$
\gamma_{\mathrm{Au}}=\frac{B}{A} \frac{\int_{\mathrm{res}} \sigma_{\mathrm{res}}^{\mathrm{Au}} d E / E}{\int_{0}^{E_{c}} M(E) \sigma_{0}{ }^{\mathrm{Au}} d E / E^{1 / 2}} .
$$

For case 2) (assume the $f$-factor $=1$ )

$$
\begin{aligned}
& \gamma_{\mathrm{U} 235}=\frac{B}{A}
\end{aligned}
$$

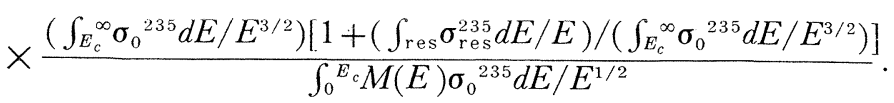

This can be written as

$$
\gamma_{\mathrm{U}^{235}}=\frac{B}{A} \frac{\int_{E_{c}{ }^{\infty} \sigma_{0}{ }^{235} d E / E^{3 / 2}[1+\rho /(1-\rho)]}}{\int_{0}{ }^{E_{c}} M(E) \sigma_{0}{ }^{235} d E / E^{1 / 2}}
$$

where

$$
\rho=\frac{\text { non- } 1 / v \text { fission rate in } \mathrm{U}^{235}}{\text { epi-Cd fission rate in } \mathrm{U}^{235}} \text {. }
$$

Now consider capture in $\mathrm{U}^{238}$. The $1 / v$ capture in Cd covered foils which must be subtracted out is

$$
\int_{E_{c}}^{\infty} d E \frac{B}{E} \frac{\sigma_{0}^{238}}{E^{1 / 2}} \equiv \mathrm{I}
$$

The sub-Cd capture is

$$
\int_{0}^{E_{c}} d E A \frac{M(E)}{E^{1 / 2}} \sigma_{0}{ }^{238} \equiv \mathrm{II} \text {. }
$$

The ratio of these is

$$
\delta \equiv \frac{\mathrm{I}}{\mathrm{II}}=\frac{B}{A} \frac{\int_{E_{c}}^{\infty} \sigma_{0}{ }^{238} d E / E^{3 / 2}}{\int_{0}^{E_{c}} M(E) \sigma_{0}{ }^{238} d E / E^{1 / 2}} .
$$

Use of (A3-5) gives

$$
\delta=\gamma_{\mathrm{Au}} \frac{\int_{0}^{E_{c}} M(E) \sigma_{0}^{\mathrm{Au}} d E / E^{1 / 2}}{\int_{\mathrm{res}} \sigma_{\mathrm{res}}^{\mathrm{Au}} d E / E} \times \frac{\int_{E_{c}}^{\infty} d E / E^{3 / 2}}{\int_{0}^{E_{c}} M(E) d E / E^{1 / 2}}
$$


or

$$
\begin{gathered}
\delta=3.2 \frac{\sigma_{0}^{\mathrm{Au}} \gamma_{\mathrm{Au}}}{\text { res. int. of } \mathrm{Au}} \quad, \quad \sigma_{0}^{\mathrm{Au}}=15.48 \text { barns }, \\
\sigma_{\mathrm{res}}^{\mathrm{Au}}=1513 \text { barns }, \\
=\frac{3.2 \times 15.48}{1513} \gamma_{\mathrm{Au}} \\
=3.27 \times 10^{-2} \gamma_{\mathrm{Au}} \\
=3.27 \times 10^{-2}\left(R_{c}-1\right)^{-1} \quad(\mathrm{~A} 3-11)
\end{gathered}
$$

where $R_{0}$ is the gold cadmium ratio discussed in sections III-E and IV-F. Use of (A3-6) gives

$$
\begin{aligned}
& \delta=\frac{\gamma_{\mathrm{U}^{235} \int_{0}^{E_{c}} M(E) \sigma_{0}{ }^{235} d E / E^{1 / 2}}}{(1-\rho)^{-1} \int_{E_{c}{ }^{\infty} \sigma_{0}{ }^{235}} d E / E^{3 / 2}} \\
& \times \frac{\int_{E_{c}{ }^{\infty} \sigma_{0}{ }^{238} d E / E^{3 / 2}}}{\int_{0}^{E_{c}} M(E) \sigma_{0}{ }^{235} d E / E^{1 / 2}} \\
& =(1-\rho) \gamma_{\mathrm{U}^{235}} \\
& =(1-\rho) \rho_{m} /\left(1-\rho_{m}\right)
\end{aligned}
$$

where

$$
\rho_{m}=\text { (measured) } \frac{\text { epi-Cd fission rate in } \mathrm{U}^{235}}{\text { total fission rate in } \mathrm{U}^{235}} .
$$

The value of $\rho$ is not precisely known, but is of the order of $\rho_{m}$ (each is approximately 0.05 ), therefore we may write

$$
\delta=\rho_{m} .
$$

We thus have two measurements from which $\delta$ can be evaluated - the Cid ratio of thin Au foils, and the epi-Cd fission fraction in $\mathrm{U}^{235}$.

The measured values of $\delta$ obtained through the two sets of measurements are compared:

$$
\begin{array}{ccc}
V_{\mathrm{H}_{2} \mathrm{O}} / V_{u} & \delta\left[=3.27 \times 10^{-2}\left(R_{0}-1\right)^{-1}\right] & \delta\left(=\rho_{m}\right) \\
1 & 0.177 & 0.144 \\
2 & .102 & .078 \\
3 & .070 & .044 \\
4 & .060 & .032
\end{array}
$$

This is the basis of the remarks in sections IV-G and $\mathrm{V}$-E that our values of $\rho_{m}$ are suspiciously low.

If the observed activities in $\mathrm{U}^{238}$ are

$$
A_{\varepsilon}=\text { epi-Cd }, A_{s}=\text { sub-Cd },
$$

these must be converted to the numbers

$$
A_{\varepsilon}^{\prime}=A_{\varepsilon}-\delta A_{s}, \quad A_{s}^{\prime}=A_{s}+\delta A_{s} .
$$

Therefore if $S=A_{\varepsilon} / A_{s}$, then

$$
\frac{A_{\varepsilon}^{\prime}}{A_{s}^{\prime}}=\frac{A_{\varepsilon}(1-\delta / S)}{A_{s}(1+\delta)}=S \frac{(1-\delta / S)}{(1+\delta)}
$$

is the ratio of interest in the $p$ measurement.

In the conversion ratio, the quantity

$$
1+\frac{A_{\varepsilon}^{\prime}}{A_{s}^{\prime}}=1+\frac{S(1-\delta / S)}{(1+\delta)}=\frac{1+S}{1+\delta}
$$

is the quantity of interest. 


\section{APPENDIX IV}

\section{Conversion Ratio Analysis}

Let

Then

$$
\begin{aligned}
\rho_{m}= & (\text { measured }) \frac{\text { epi-Cd fission rate in } 25}{\text { total fission rate in } 25}, \\
\rho= & \frac{\text { non- } 1 / v \text { fission rate in } 25^{*}}{\text { epi-Cid fission rate in } 25}, \\
\rho_{0}= & \frac{\text { non- } 1 / v \text { fission rate in } 25}{\text { thermal (i.e., } 1 / v) \text { fission rate in } 25}, \\
\rho_{0}= & \rho \rho_{m} /\left(1-\rho \rho_{m}\right), \\
& \rho_{m} \approx 0.05, \\
& \rho \approx 0.05, \\
& \rho_{0} \approx 0.0025, \\
S= & \frac{\text { epi-Cd capture rate in } 28}{\text { sub-Cd capture rate in } 28} .
\end{aligned}
$$

and

$$
1+S=\frac{\text { total capture rate in } 28}{\text { sub-Cd capture rate in } 28}
$$

$$
\frac{1+S}{1+\delta}=\frac{\text { total capture rate in } 28}{\text { thermal }(1 / v) \text { capture rate in } 28}
$$

(See Appendix III).

Now

$C=\frac{\text { total capture in } 28}{\text { total absorption in } 25}$

$$
\begin{aligned}
& =\frac{\text { total capture in } 28}{\left(1+\alpha_{\mathrm{th}}\right) \text { thermal fission in } 25+\left(1+\alpha_{f}\right)(\text { non-thermal fission in 25) }} \\
& =\frac{\text { total capture in } 28}{\text { thermal fission in } 25\left[\left(1+\alpha_{\mathrm{th}}\right)+\rho_{0}\left(1+\alpha_{f}\right)\right]} \\
& =\frac{(1+S)}{(1+\delta)} \frac{\text { thermal capture in } 28}{\text { thermal fission in } 25}\left[\left(1+\alpha_{\mathrm{th}}\right)\left(1+\frac{1+\alpha_{f}}{1+\alpha_{\mathrm{th}}} \rho_{0}\right)\right]^{-1} \\
& =\frac{(1+S)}{(1+\delta)} \frac{\sum_{28}^{\text {abs }} / \sum_{\mathrm{fue}}^{\text {abs }}}{\sum_{25}^{\mathrm{fission}} / \Sigma_{\mathrm{fuel}}^{\text {abs }}}\left[\left(1+\alpha_{\mathrm{th}}\right)\left(1+\frac{1+\alpha_{f}}{1+\alpha_{\mathrm{th}}} \rho_{0}\right)\right]^{-1} \\
& =\frac{(1+S)}{(1+\delta)} \frac{\sum^{28}}{\eta / \nu_{25}}\left[\left(1+\alpha_{\mathrm{th}}\right)\left(1+\frac{1+\alpha_{f}}{1+\alpha_{\mathrm{th}}} \frac{\rho \rho_{m}}{1-\rho \rho_{m}}\right)\right]^{-1} \\
& =\frac{(1+S)}{(1+\delta)} \frac{\nu_{25} \Sigma^{28}}{\eta\left(1+\alpha_{\mathrm{th}}\right)\left[1+\beta \rho \rho_{m} /\left(1-\rho \rho_{m}\right)\right]} ; \quad \Sigma^{28}=\sum_{28}^{\text {abs }} / \sum_{\mathrm{fuel}}^{\mathrm{abs}}, \quad \beta=\frac{1+\alpha_{f}}{1+\alpha_{\mathrm{th}}} \cong 1 .
\end{aligned}
$$

*See footnote to appendix III. 
On the other hand,

$$
\begin{aligned}
\mathrm{C} & =\frac{(1+S) \times \text { sub-Cd capture rate in } 28}{\left(1+\alpha_{\mathrm{th}}\right) \text { sub-Cd fission in } 25+\text { epi-Cd absorption in } 25} \\
& =\frac{(1+S) \times \text { sub-Cd capture in } 28}{\left(1+\alpha_{\mathrm{th}}\right)(\text { sub-Cd fission in } 25+\text { epi-Cd } 1 / v \text { fission in } 25)+\left(1+\alpha_{f}\right) \text { epi-Cd non- } 1 / v \text { fission in } 25} \\
& =\frac{(1+S) \text { sub-Cd capture in } 28}{\left(1+\alpha_{\mathrm{th}}\right) \text { sub-Cd fission in } 25\left(1+\frac{\text { epi-Cd } 1 / v \text { fission in } 25}{\text { sub-Cd fission in } 25}+\beta \frac{\text { non- } 1 / v}{\text { sub-Cd }}\right)} .
\end{aligned}
$$

From the definitions of $\rho, \rho_{m}$, and $\rho_{0}$, the bracketed term in the denominator is

$$
1+\frac{\rho_{m}(1-\rho)}{1-\rho_{m}}+\beta \frac{\rho \rho_{m}}{1-\rho \rho_{m}}=\frac{1+\rho \rho_{m}(\beta-1)}{1-\rho_{m}}
$$

or

$$
C=\frac{(1+S) \nu_{25} \Sigma^{28}}{\eta\left(1+\alpha_{\mathrm{th}}\right)\left[1+\rho \rho_{m}(\beta-1)\right] /\left(1-\rho_{m}\right)} .
$$

These two expressions for $C$ must agree, therefore

or

$$
(1+\delta)\left(1+\frac{\beta \rho \rho_{m}}{1-\rho \rho_{m}}\right)=\frac{1+\rho \rho_{m}(\beta-1)}{1-\rho_{m}}
$$

or

$$
(1+\delta) \frac{1+\rho \rho_{m}(\beta-1)}{1-\rho \rho_{m}}=\frac{1+\rho \rho_{m}(\beta-1)}{1-\rho_{m}}
$$

$$
\begin{aligned}
\frac{(1+\delta)}{1-\rho \rho_{m}} & =\frac{1}{1-\rho_{m}}, \\
1+\delta & =\frac{1-\rho \rho_{m}}{1-\rho_{m}}, \\
\delta & =\frac{1-\rho \rho_{m}-\left(1-\rho_{m}\right)}{1-\rho_{m}} \\
& =\frac{\rho_{m}(1-\rho)}{\left(1-\rho_{m}\right)} .
\end{aligned}
$$

From the point of view of the definition of $\delta$ the same result follows, as is shown in Appendix III.

For calculating $C$, note that $\beta \cong 1$ and $\rho \rho_{m}<<1$. Then either:

or

$$
C=\frac{(1+S) \nu_{25} \Sigma^{28}}{(1+\delta)\left(1+\alpha_{\mathrm{th}}\right) \eta}, \quad \text { denote by } C_{\delta}
$$

$$
C=\frac{(1+S) \nu_{25} \Sigma^{28}}{\eta\left(1+\alpha_{\mathrm{th}}\right) /\left(1-\rho_{m}\right)}, \text { denote by } C_{\rho_{m}} .
$$

If $C_{0}=\nu_{25} \Sigma^{28} / \eta\left(1+\alpha_{\mathrm{th}}\right)$, then

$$
C_{\delta}=C_{0}(1+S) /(1+\delta) \quad \text { and } \quad C_{\rho_{m}}=C_{0}(1+S)\left(1-\rho_{m}\right) ;
$$

$1+\alpha_{25}=1.192, \nu_{25}=2.47, \eta_{\mathrm{nat}}=1.309$. The values of $C_{0}$ for the various enrichments are

$$
\begin{array}{ll}
1.0 \% & C_{0}=0.3941 \\
1.15 \% & C_{0}=0.3538 \\
1.3 \% & C_{0}=0.3109
\end{array}
$$




\begin{tabular}{llrrrrr} 
& & $1+S$ & $1+\delta$ & $1-\rho_{m}$ & \multicolumn{1}{c}{$C_{\delta}$} & $C_{\rho_{m}}$ \\
$1 \%$ & 1 & 3.00 & 1.177 & 0.856 & 1.005 & 1.012 \\
& 2 & 2.01 & 1.102 & .922 & 0.719 & 0.730 \\
& 3 & 1.79 & 1.070 & .956 & .659 & .674 \\
& 4 & 1.60 & 1.060 & .968 & .595 & .610 \\
$1.15 \%$ & 2 & 2.20 & 1.102 & .918 & .706 & .715 \\
& 3 & 1.93 & 1.070 & .946 & .638 & .646 \\
$1.3 \%$ & 1.5 & 2.80 & 1.139 & .846 & .764 & .736 \\
& 2 & 2.24 & 1.102 & .884 & .632 & .616 \\
& 3 & 1.89 & 1.070 & .938 & .549 & .551
\end{tabular}




\section{APPENDIX V}

\section{Derivation of Equation 17}

The following is based on a BNL internal memorandum by H. J. Kouts, April 1954.

1. Begin with 1 virgin neutron from thermal fission.

2. It has a probability $P_{f}$ of causing fast fission.

3 . It has a probability $P_{f}\left(1+\alpha_{28}\right)$ of being absorbed above the $\mathrm{U}^{238}$ fission threshold.

4 . It has a probability $P_{f} \alpha_{28}$ of being captured in $\mathrm{U}^{238}$.

5. It has a probability $\left[1-P_{f}\left(1+\alpha_{28}\right)\right]$ of slowing down past the 28 fission threshold.

6 . Therefore, the total number of neutrons slowing down past the 28 threshold is

$$
\begin{aligned}
\varepsilon & =1-P_{f}\left(1+\alpha_{28}\right)+\nu_{28} P_{f}\left[1-P_{f}\left(1+\alpha_{28}\right)+\nu_{28} P_{f}(1-\ldots)\right] \\
& =\frac{\varepsilon-\left[1-P_{f}\left(1+\alpha_{28}\right)\right]}{\nu_{28} P_{f}}
\end{aligned}
$$

or

$$
P_{f}=\frac{\varepsilon-1}{\varepsilon \nu_{28}-1-\alpha_{28}} .
$$

7. The total number of fast captures is

$$
\begin{aligned}
\mathcal{N}_{f} & =P_{f} \alpha_{28}+\nu_{28} P_{f} \cdot P_{f} \alpha_{28}+\ldots \\
& =\frac{P_{f} \alpha_{28}}{1-\nu_{28} P_{f}} .
\end{aligned}
$$

Therefore

$$
P_{f}=\frac{\mathcal{N}_{f}}{\alpha_{28}+\nu_{28} \mathcal{N}_{f}}
$$

From (A5-1) and (A5-2),

$$
\mathcal{N}_{f}=\frac{(\varepsilon-1) \alpha_{28}}{\nu_{28}-1-\alpha_{28}} \text {. }
$$

8. Let $\mathcal{L}_{1}$ be the non-leakage probability in the fast region, then $\varepsilon \mathcal{L}_{1}$ neutrons reach the resonance region.

9. $\varepsilon \mathcal{L}_{1} p$ avoid resonance capture.

10. $\varepsilon \mathcal{L}_{1}(1-p)$ are captured in the resonance region.

11. Let $\mathcal{L}_{2}$ be the non-leakage probability in the resonance region, then $\varepsilon \mathcal{L}_{1} \mathcal{L}_{2} p$ neutrons reach thermal.

12. Let $\mathcal{L}_{3}$ be the non-leakage probability in the thermal region, then $\varepsilon \mathcal{L}_{1} \mathcal{L}_{2} \mathcal{L}_{3} p f$ neutrons are absorbed in uranium.

13. $\varepsilon \mathcal{L}_{1} \mathcal{L}_{2} \mathcal{L}_{3}$ pf $\Sigma$ are absorbed in $\mathrm{U}^{238}$ at thermal where $\Sigma=\Sigma_{a}{ }^{238} /\left(\Sigma_{a}{ }^{238}+\Sigma_{a}{ }^{235}\right)$.

In Appendix III it is shown that the ratio of the epi-thermal (resonance + fast) capture rate to thermal capture rate in $\mathrm{U}^{238}$ is given by

$$
\frac{S(1-\delta / S)}{1+\delta} \text {. }
$$

Therefore, from (A5-3) and steps 10 and 13:

$\frac{S(1-\delta / S)}{1+\delta}=\frac{\varepsilon \mathcal{L}_{1}(1-p)+(\varepsilon-1) \alpha_{28} /\left(\nu_{28}-1-\alpha_{28}\right)}{\varepsilon \mathcal{L}_{1} \mathcal{L}_{2} \mathcal{L}_{3} p f \Sigma}$,

from which Equation 17 follows by solving for $p$. 


\section{APPENDIX VI}

\section{Three-Group Analysis of Fast Leakage}

Assume the group equations in the form

Fast:

$$
D_{1} \nabla^{2} \phi_{1}-\Sigma_{1} \phi_{1}+f \eta \Sigma_{s} \phi_{s}+\mu \nu_{28} \Sigma_{1} \phi_{1}=0
$$

Intermediate:

$$
D_{2} \nabla^{2} \phi_{2}-\Sigma_{2} \phi_{2}+[1-\mu(1+\alpha)] \Sigma_{1} \phi_{1}=0,
$$

Thermal:

$$
D_{s} \nabla^{2} \phi_{s}-\Sigma_{\mathrm{s}} \phi_{\mathrm{s}}+p \Sigma_{2} \phi_{2}=0
$$

where $\mu$ is that fraction of the fast removal cross section which leads to fast fission, and $\alpha$ is an average value for $\mathrm{U}^{238}$ in the fast fission region. The usual assumption of identical space variation of the separate group fluxes and of a buckling leads to the simplification

$$
\begin{gathered}
-\left(A_{1}-\mu \nu_{28}\right) \Sigma_{1} \phi_{1}+f \eta \Sigma_{s} \phi_{s}=0, \\
-A_{2} \Sigma_{2} \phi_{2}+[1-\mu(1+\alpha)] \Sigma_{1} \phi_{1}=0, \\
-A_{s} \Sigma_{s} \phi_{s}+p \Sigma_{2} \phi_{2}=0,
\end{gathered}
$$

where the $A_{i}$ are the reciprocals of the non-leakage probabilities $\mathcal{L}_{i}$ :

$$
A_{i}=\left(1+L_{i}{ }^{2} B^{2}\right)=\mathcal{L}_{i}{ }^{-1} .
$$

The secular equation (critical equation) of the set (1) is*

$$
A_{1} A_{2} A_{s}=\frac{1-\mu(1+\alpha)}{1-\mu \nu_{28} \mathcal{L}_{1}} f \eta p
$$

therefore the finite lattice value of $\varepsilon$ is

$$
\varepsilon=\frac{1-\mu(1+\alpha)}{1-\mu \nu_{28} \mathcal{L}_{1}}
$$

The quantity measured in the experiments is $F$; it has been defined in section III-F, and in terms of the three-group parameters it is

$$
F=\frac{\mu \Sigma_{1} \phi_{1}}{f \eta \Sigma_{s} \phi_{s} / \nu_{25}}
$$

* Equation (33) of the text differs from Equation (A6-2) by including effects of resonance fissions in $\mathrm{U}^{235}$.
However, by (1a)

$$
\frac{\Sigma_{1} \phi_{1}}{\sum_{s} \phi_{s}}=\frac{f \eta}{A_{1}-\mu \nu_{28}}
$$

hence (4) and (5) lead to

and

$$
F=\frac{\mu \nu_{25}}{A_{1}-\mu \nu_{28}}
$$

$$
\mu=\frac{F A_{1}}{F \nu_{28}+\nu_{25}}
$$

On substitution of (7), Equation (3) becomes

$$
\varepsilon=1+F \frac{\nu_{28}-A_{1}(1+\alpha)}{\nu_{25}} .
$$

An infinite lattice would be characterized by $A_{1}=1$. Equation (8) would in this case become

$$
\varepsilon_{\infty}=1+F_{\infty} \frac{\nu_{28}-(1+\alpha)}{\nu_{25}}
$$

where, by Equation (6)

$$
F_{\infty}=\frac{\mu \nu_{25}}{1-\mu \nu_{28}} .
$$

From Equations (6), (7), and (10), it follows that

$$
\frac{F}{F_{\infty}}=\mathcal{L}_{1}-F \frac{\nu_{28}}{\nu_{25}}\left(1-\mathcal{L}_{1}\right) \cong \mathcal{L}_{1}
$$

Also, from Equations (8) and (9),

$$
\begin{gathered}
(\varepsilon-1)=\left[\frac{F}{F_{\infty}} \frac{\nu_{28}-A_{1}(1+\alpha)}{\nu_{28}-(1+\alpha)}\right]\left[F_{\infty} \frac{\nu_{28}(1+\alpha)}{\nu_{25}}\right] \\
\cong \mathcal{L}_{1} C(\varepsilon-1)_{\infty}
\end{gathered}
$$

where

$$
C=\frac{\nu_{28}-A_{1}(1+\alpha)}{\nu_{28}-(1+\alpha)}=1-\frac{\left(A_{1}-1\right)(1+\alpha)}{\nu_{28}-(1+\alpha)} .
$$

Table 11 contains values of

$$
(\varepsilon-1)=F \frac{\nu_{28}-(1+\alpha)}{\nu_{25}} .
$$

Multiplication of these by $A_{1}$ will (by Equation 11 above) lead to $(\varepsilon-1)_{\infty}$. On the other hand, multi- 
plying them by $C$ yields the values which apply in a finite lattice.

A simpler expression for the relation between $\varepsilon$ and $\varepsilon_{\infty}$ can be found from direct substitution of
(7) into (3) and into its corresponding infinite lattice form:

$$
\frac{\varepsilon}{\varepsilon_{\infty}}=1-F\left(A_{1}-1\right) \frac{\nu_{28}}{\nu_{25}} .
$$

